\title{
Application of immunohistochemistry in diagnosis and management of malignant mesothelioma
}

\author{
David B. Chapel ${ }^{1,2}$, Jefree J. Schulte ${ }^{1}$, Aliya N. Husain ${ }^{1}$, Thomas Krausz ${ }^{1}$ \\ ${ }^{1}$ Department of Pathology, University of Chicago, Chicago, IL 60637, USA; ${ }^{2}$ Department of Pathology, Brigham and Women's Hospital, Boston, \\ MA 02115 , USA \\ Contributions: (I) Conception and design: DB Chapel, T Krausz; (II) Administrative support: None; (III) Provision of study materials or patients: \\ None; (IV) Collection and assembly of data: DB Chapel; (V) Data analysis and interpretation: DB Chapel; (VI) Manuscript writing: All authors; (VII) \\ Final approval of manuscript: All authors. \\ Correspondence to: David B. Chapel, MD. Brigham and Women's Hospital, 75 Francis Street, Department of Pathology, Amory 3, Boston, MA 02115, \\ USA. Email: dchapel@bwh.harvard.edu.
}

\begin{abstract}
Immunohistochemistry plays an indispensable role in accurate diagnosis of malignant mesothelioma, particularly in morphologically challenging cases and in biopsy and cytology specimens, where tumor architecture is difficult or impossible to evaluate. Application of a targeted panel of mesothelialand epithelial-specific markers permits correct identification of tumor lineage in the vast majority of cases. An immunopanel including two mesothelial markers (calretinin, CK5/6, WT-1, or D2-40) and two epithelial markers (MOC-31 and claudin-4) offers good sensitivity and specificity, with adjustments as appropriate for the differential diagnosis. Once mesothelial lineage is established, malignancy-specific studies can help verify a diagnosis of malignant mesothelioma. BAP1 loss, CDKN2A homozygous deletion, and MTAP loss are highly specific markers of malignancy in a mesothelial lesion, and they attain acceptable diagnostic sensitivity when applied as a diagnostic panel. Novel markers of malignancy, such as 5-hmC loss and increased EZH2 expression, are promising, but have not yet achieved widespread clinical adoption. Some diagnostic markers also have prognostic significance, and PD-L1 immunohistochemistry may predict tumor response to immunotherapy. Application and interpretation of these immnuomarkers should always be guided by clinical history, radiographic findings, and above all histomorphology.
\end{abstract}

Keywords: Immunohistochemistry; mesothelioma; BAP1; peritoneum; pleura

Submitted Oct 18, 2019. Accepted for publication Nov 20, 2019.

doi: $10.21037 /$ tlcr.2019.11.29

View this article at: http://dx.doi.org/10.21037/tlcr.2019.11.29

\section{Introduction}

A spectrum of hyperplastic and neoplastic mesothelial lesions arises from the serosal linings of the pleura, pericardium, and peritoneum, and tunica vaginalis. Mesothelial neoplasms range from benign localized tumors to aggressive diffuse malignancies that destructively infiltrate surrounding tissues and can metastasize to distant sites. Approximately $85 \%$ of malignant mesotheliomas arise in the pleural cavity, with most of the remainder arising in the peritoneum (1). Paratesticular and primary pericardial malignant mesotheliomas are well-documented but rare tumors. Malignant mesothelioma has a poor prognosis, with median survival of 16 months for pleural tumors in one recent large study (2). Accordingly, accurate distinction from benign mesothelial proliferations is crucial, to avoid both undue psychic injury and unnecessary surgical or systemic therapies.

Accurate diagnosis of mesothelial proliferations, however, is one of surgical pathology's persistent challenges. Malignant mesothelioma is a rare tumor, with approximately 2,500 new cases diagnosed annually in the United States (3). Furthermore, morphologic overlap between benign mesothelial proliferations and malignant mesothelioma 


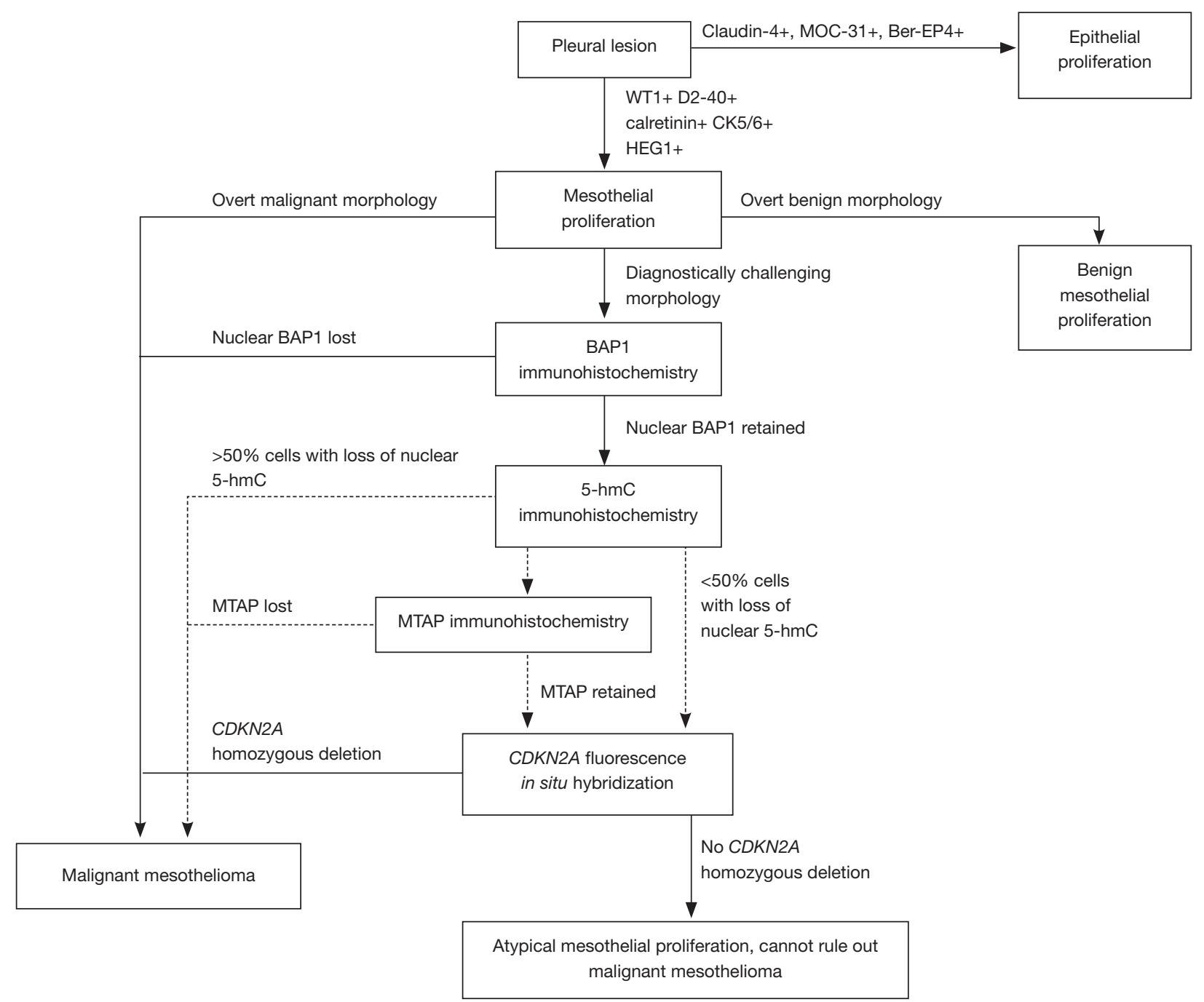

Figure 1 Algorithm for establishing a diagnosis of malignant mesothelioma. Dashed lines indicate provisional diagnostic markers not yet in widespread use. Adapted from Chapel DB, et al. Mod Pathol 2019;32:376-86.

complicates reliable distinction by histomorphology alone, particularly in small biopsy and cytopathology specimens. In consequence, ancillary diagnostic techniques, particularly immunohistochemical stains, have become indispensable for accurate diagnosis of mesothelioma. Nonetheless, it warrants emphasis that careful morphologic examination must precede application of ancillary diagnostic studies, and that the results of these studies must be interpreted in the context of the pathologist's morphologic impression.

The landscape of immunohistochemical studies for diagnosis of mesothelial proliferations has evolved rapidly in the last decade, including the introduction of some immunostains that can also be applied in evaluation of non- mesothelial tumors. Herein, we begin with a discussion of immunomarkers useful for establishing mesothelial lineage, followed by a detailed handling of markers useful for distinguishing benign from malignant mesothelial lesions. Prognostic and predictive markers are also addressed. We conclude with discussion of rare tumor sites and presentations.

\section{Establishing mesothelial lineage}

Establishing lineage is the first step in diagnosis of a serosa-based tumor (Figure 1). A carefully selected panel of mesothelial and epithelial immunomarkers permits 
confident lineage identification in virtually all cases with sufficient tissue for evaluation, bearing in mind that rare non-epithelial tumors may also occur in the serosa and mimic mesothelioma (4). Recent guidelines recommend using mesothelial and epithelial markers with sensitivity and specificity of at least $80 \%$ (4), although in practice, markers with even higher specificity are available and are preferred.

Both our experience and the published literature suggest that markers of mesothelial and epithelial lineage show equivalent sensitivity in the pleura and peritoneum $(4,5)$. However, because the differential diagnostic considerations in these two sites may differ substantially, mesothelial markers may show different specificity in the pleura versus peritoneum, due to cross-reactivity of certain markers in the most common carcinomas at each site. These caveats are handled in a recent review (6) and consensus guidelines (4), and are discussed alongside each marker below.

Panels of mesothelial- and epithelial-specific immunomarkers can be applied to large resections, small biopsies, and cell blocks. Current guidelines recommend that a cutoff of $10 \%$ staining should be used for cytoplasmic and membranous markers (4). It should be emphasized that (with the rare exceptions) the markers discussed in this section demonstrate mesothelial lineage only, and do not generally distinguish benign from malignant mesothelial lesions, which instead relies on clinical and radiologic correlation, careful morphologic assessment, and application of additional ancillary studies, as discussed below. Reported sensitivity and specificity statistics for the discussed markers are summarized in Table 1.

\section{Markers of mesothelial lineage}

Calretinin has long been considered a reliable marker for establishing mesothelial lineage. We encounter calretinin immunostains frequently in consultation and use this marker routinely in-house. Dual cytoplasmic and strong nuclear calretinin expression supports mesothelial lineage (Figure 2). Other staining patterns, especially lack of nuclear staining, have lower specificity and should be interpreted cautiously. When polyclonal antibodies against human recombinant calretinin are used, calretinin is $80-100 \%$ sensitive for epithelioid malignant mesothelioma (7-26), although variation in calretinin antibodies in clinical use may account for imperfect sensitivity in practice (12,27-29). Furthermore, sensitivity is somewhat lower in sarcomatoid mesotheliomas, with reported figures ranging from 5-88\%, but generally in the range of 50-60\% (11-13,27,28,30-34).
Calretinin is not entirely specific for mesothelial cells, with expression reported in a substantial subset of breast carcinomas, squamous cell carcinomas, and serous ovarian carcinomas $(8-10,35,36)$, and in $10-60 \%$ of sarcomatoid carcinomas $(30,34,37,38)$. Calretinin is reported in only $0-10 \%$ of pulmonary adenocarcinomas and renal cell carcinomas $(7,11,26,39)$. Other calretinin-positive lesions, such as ovarian sex cord-stromal tumors and adrenocortical tumors, may rarely enter the clinical and morphologic differential diagnosis for mesothelial proliferations of the peritoneum. Therefore, although generally reliable and widely used, calretinin is an imperfect mesothelial marker that should be interpreted in the context of a broader immunopanel.

CK5/6 is another widely used mesothelial immunomarker. Like calretinin, CK5/6 shows good but imperfect sensitivity in practice, ranging from 51-100\% $(8,9,11,15-18,20,21,23,24,26,29,35,38,40)$. In sarcomatoid mesothelioma, however, sensitivity is low, ranging from $13-29 \%(6,11,33,34,41)$. This marker is best studied in the differential diagnosis with adenocarcinoma of the lung, of which $0-20 \%$ (but generally fewer than $5 \%$ ) are positive $(24,38,40,42,43)$. CK5/6 is expressed by squamous cell carcinomas (including poorly differentiated squamous cell tumors lacking overt squamous morphology), ovarian serous carcinomas, breast carcinoma (particularly basallike tumors), and sarcomatoid carcinomas of the lung $(8,9,23,24,34,35,38,44)$. Accordingly, our use of CK5/6 is generally limited to cases where adenocarcinoma of the lung is effectively the only entity on the differential diagnosis.

WT- 1 has reported sensitivity ranging from $70-100 \%$ for establishing mesothelial lineage $(8,9,11,15-$ $17,21,24,26,35,38)$, and just $10-45 \%$ for sarcomatoid mesothelioma (6,11,30,32-34). A clean nuclear staining pattern permits easy interpretation, and WT-1 expression is virtually zero in both squamous cell carcinoma and adenocarcinoma of the lung and in renal cell carcinoma $(8,38,40,45)$. Although few studies report WT-1 expression in up to $37 \%$ of triple-negative breast carcinomas (43), the preponderance of evidence suggests that WT-1 is expressed in at most a few percent of breast cancers, and is typically weak and focal $(26,35,46-48)$. Importantly, WT-1 is a classic marker of both low-grade and high-grade serous carcinomas of the uterine adnexa, which limits its usefulness in peritoneal tumors, where mesothelioma and serous carcinomas may also show considerable morphologic overlap.

Podoplanin (frequently termed "D2-40", in reference 
Table 1 Sensitivity and specificity of mesothelial- and epithelial-specific markers

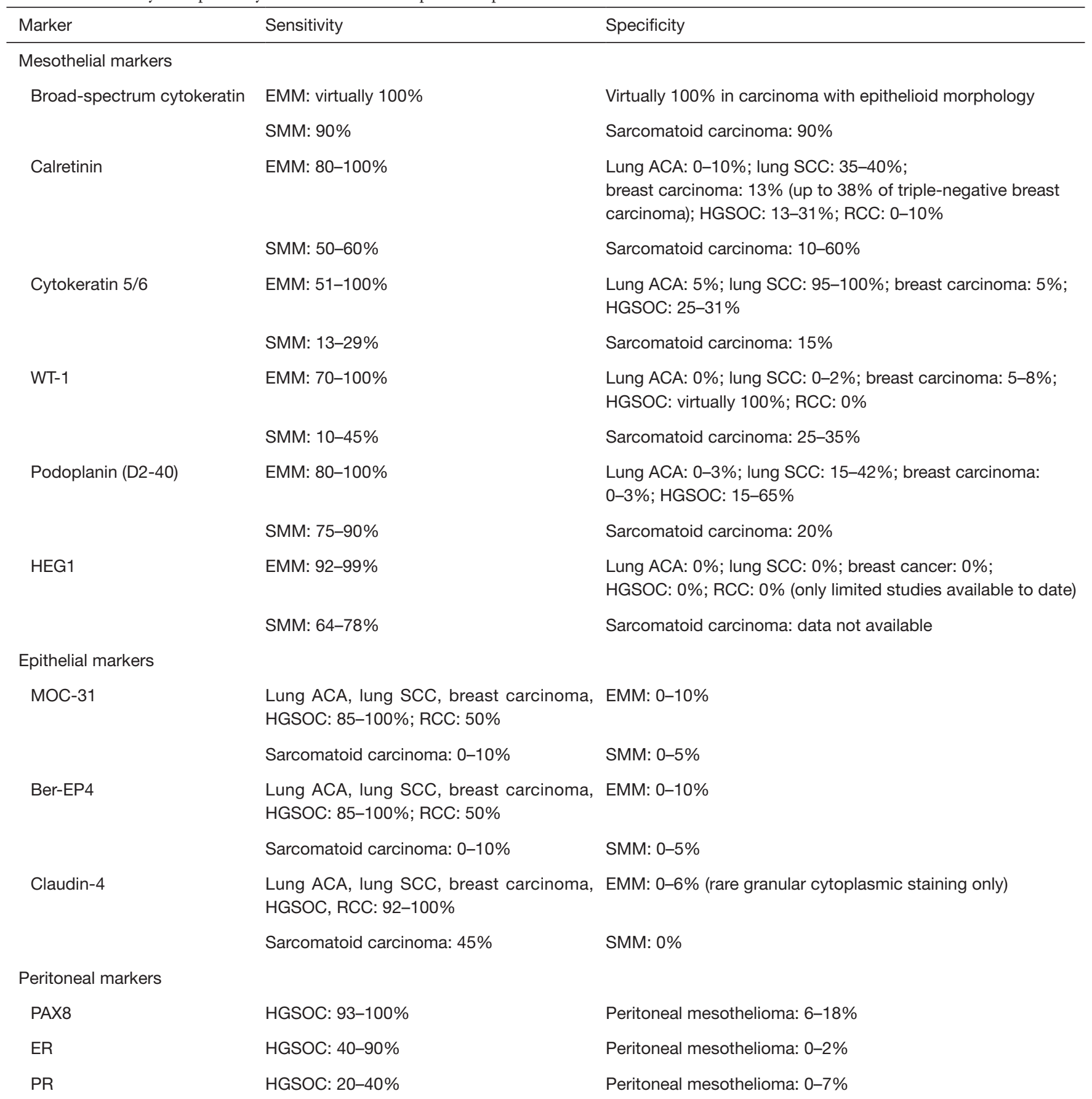

Where sufficient data were available to confidently identify outliers, they have been excluded from this table, but they are noted in the manuscript text. ACA, adenocarcinoma; Ca, carcinoma; EMM, epithelioid malignant mesothelioma; ER, estrogen receptor; HGSOC, high-grade serous ovarian carcinoma; PR, progesterone receptor; RCC, renal cell carcinoma; SCC, squamous cell carcinoma; SMM, sarcomatoid malignant mesothelioma. 

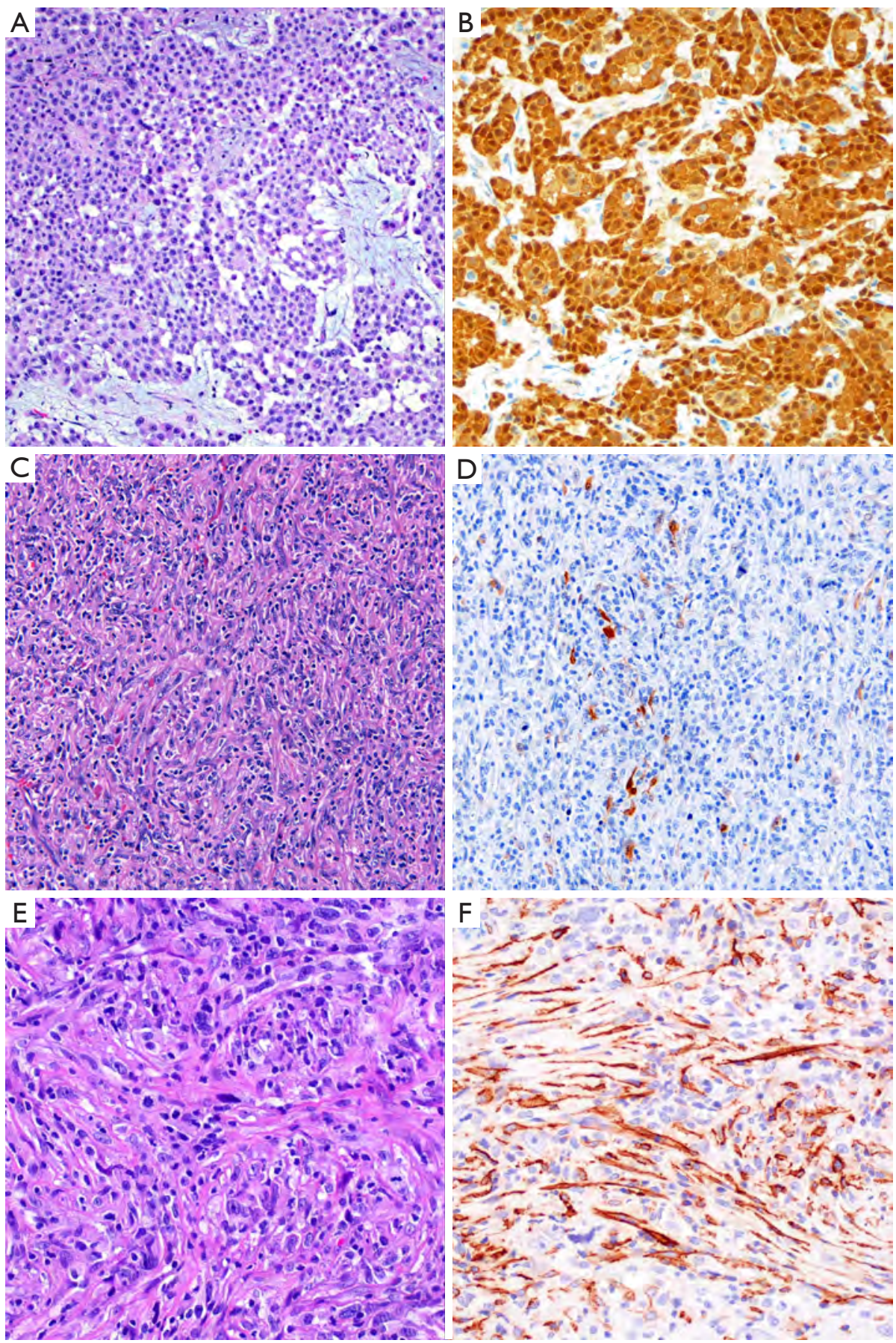

Figure 2 Mesothelial immunomarkers are useful for establishing tumor lineage. (A,B) Strong and diffuse cytoplasmic-and-nuclear calretinin staining in an epithelioid malignant mesothelioma (A, hematoxylin and eosin, original magnification $\times 100$; B, calretinin immunostain, original magnification $\times 200)$. (C,D) Focal single-cell calretinin staining in a sarcomatoid malignant mesothelioma (C, hematoxylin and eosin, original magnification 200×; D, calretinin immunostain, original magnification $\times 200$ ). (E,F) Diffuse membranous D2-40 (podoplanin) staining in a sarcomatoid malignant mesothelioma (E, hematoxylin and eosin; F, D2-40 immunostain; original magnification $\times 200)$. 
to the most widely used anti-podoplanin clone) is a sensitive marker of epithelioid mesothelial lesions, showing membranous staining in $80-100 \%(8,9,15,16,19,24,26$, $35,38,49,50)$ (Figure 2). Despite some discouraging older data, podoplanin also appears to be a sensitive marker for sarcomatoid mesothelioma, with staining in up to $90 \%$ of tumors $(13,28,32,34,37,49,50)$, although careful examination is required, since D2-40 staining of lymphatic spaces may mimic tumor staining (4). Podoplanin is imperfectly specific for mesothelioma. It is expressed in 13$65 \%$ of serous ovarian carcinomas and approximately half of squamous cell carcinomas $(8,9,44,50)$. True membranous staining is virtually always negative in conventional lung adenocarcinoma, but is reported to be seen in $25-30 \%$ of sarcomatoid carcinoma $(32,34,49)$. Additionally, strong podoplanin is classic for certain non-mesothelial tumors, including seminoma and angiosarcoma, which may rarely enter into the differential diagnosis with mesothelial proliferations.

Heart development protein with EGF-like domains 1 (HEG1) is a recently described mesothelial marker $(5,33)$. Based on two clinically oriented studies of HEG1, cytoplasmic-membranous staining for HEG1 is reportedly $92-99 \%$ sensitive and $83-99 \%$ specific for mesothelial lineage overall, with HEG1 showing higher sensitivity and specificity than calretinin, D2-40 and WT-1 immunohistochemistry in both studies $(5,33)$. Furthermore, HEG1 is reportedly 100\% specific for epithelioid mesothelioma in the differential diagnosis with pulmonary adenocarcinoma (although 4 of 7 pleomorphic carcinomas were positive for HEG1), and 64-78\% sensitive for sarcomatoid mesothelioma $(5,33)$. As of this writing, the HEG1 antibody is currently commercially available only in Japan, although we anticipate that it will become more widely available in the near future.

Other markers that are reportedly useful for establishing mesothelial lineage include mesothelin, thrombomodulin, caveolin, tenascin-X, and type III collagen. We do not currently use these markers in our practice, but they have been recently reviewed elsewhere (6).

\section{Markers of epithelial lineage}

Epithelial markers should also be included alongside mesothelial markers when evaluating lineage of pleural or peritoneal lesions. Many markers are available, and the landscape of epithelial markers has, with few exceptions discussed here, remained largely unchanged over the last
20 years. Some older epithelial markers - such as CEA, CD15, BG8, and B72.8 (also termed TAG-72 or BRST3)may seem somewhat old-fashioned, but they remain accurate tools for distinction of epithelial and mesothelial lesions (7,15-22,24,26,29,40,51,52), although sensitivity for sarcomatoid carcinoma is poor (32).

Two antibodies against the epithelial cell adhesion molecular (Ep-CAM) remain in widespread use: BerEP4 and MOC-31. Positive staining for these markers is cytoplasmic with membranous accentuation, although strong staining may simply appear cytoplasmic. Both MOC-3 1 and Ber-EP4 are generally reported to be 85-100\% sensitive for adenocarcinoma and squamous cell carcinoma of the lung, adenocarcinoma of the breast, and serous ovarian carcinoma $(7,8,14,19-21,23,24,26,40,52-55)$. Conversely, they mark only half of renal cell carcinomas, limiting their usefulness in this differential diagnosis (11). Furthermore, because Ep-CAM is lost in cells with sarcomatoid differentiation, MOC-31 and Ber-EP4 are poorly sensitive for sarcomatoid carcinoma, with reported sensitivity from $0-30 \%$ (though generally in the $0-10 \%$ range) $(4,28,32,34)$.

Ber-EP4 shows 60-100\% specificity for epithelial lineage in the differential diagnosis with mesothelial tumors, with reported figures typically in the range of $90-100 \%(7,8,14,15,18-21,23,25,52-56)$. Similarly, although occasional studies have reported up to $30 \%$ of mesotheliomas with some MOC-31 expression (28), MOC-31 is generally reported to be $90-100 \%$ specific for epithelial lineage $(8,20,21,24,55)$, particularly when only strong and diffuse staining is considered to support epithelial lineage (20) (Figure 3). Still, we have occasionally encountered bona fide mesotheliomas with strong and diffuse MOC-31 and/or Ber-EP4 staining, highlighting the value of an immunopanel that also includes at least two mesothelial markers and claudin-4.

Indeed, there appears to be growing consensus that claudin-4 is the best epithelial marker currently in diagnostic use (4,53-57). Claudin-4 is a component of epithelial tight junctions, and membranous staining for claudin-4 is up to $92-100 \%$ sensitive and $94-100 \%$ specific for epithelial lineage in the differential diagnosis with mesothelial lesions (14,16,24,35,53-59) (Figure 3), and rare mesotheliomas with claudin-4 staining show only focal, granular cytoplasmic positivity ( $<10 \%$ of tumor cells) (16). Claudin-4 is highly accurate in effusion cytology specimens, as well $(54,55,59)$. Although sensitivity of claudin-4 remains high in poorly differentiated adenocarcinoma of the 

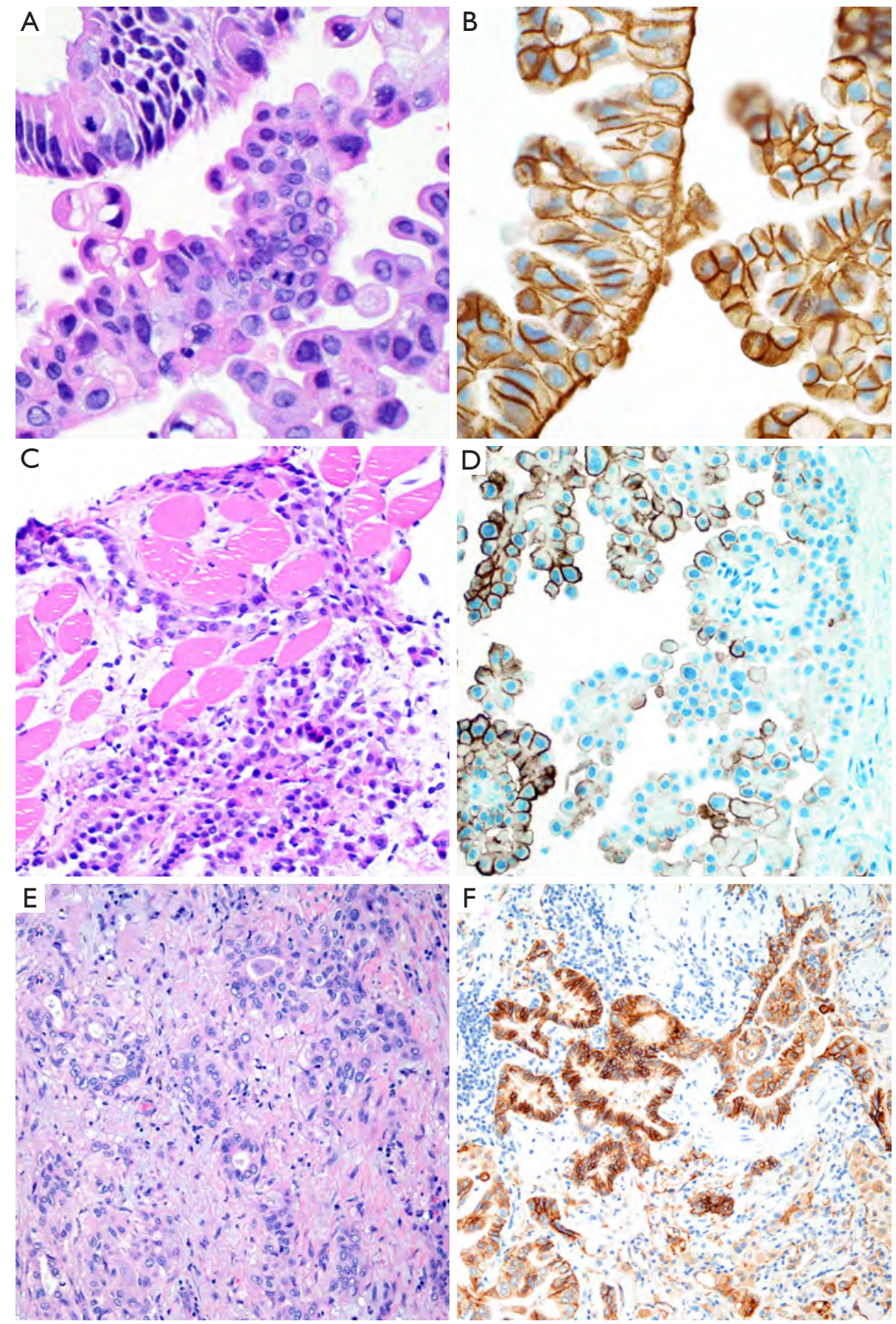

Figure 3 Epithelial immunomarkers are useful for establishing tumor lineage. (A,B) Strong and diffuse membranous MOC-31 staining in an adenocarcinoma of the lung (A, hematoxylin and eosin; B, MOC-31 immunostain; original magnification $\times 400)$. (C,D) Although most mesothelial lesions are negative for MOC-31, they may occasionally show patchy MOC-31 staining, as in this epithelioid malignant mesothelioma (C, hematoxylin and eosin; D, MOC-31 immunostain; original magnification $\times 200)$. (E,F) Strong and diffuse membranous claudin-4 staining in an adenocarcinoma of the lung (E, hematoxylin and eosin; F, claudin-4 immunostain; original magnification $\times 200)$. 
lung (56), sensitivity is decreased in frankly sarcomatoid carcinomas, with one study reporting $45 \%$ sensitivity and $100 \%$ specificity of claudin-4 expression for sarcomatoid carcinoma of the lung (37).

In our diagnostic practice, we routinely employ a panel of two to three mesothelial and two epithelial markers, typically calretinin, WT-1 and/or D2-40, MOC-31, and claudin-4 (4). The first four markers are widely available. Although not all pathology practices have adopted the claudin-4 immunostain, evidence suggests that this excellent epithelial marker should be used in challenging cases, including by send-out testing or expert consultation. In our consultation practice, we review all epithelial and mesothelial markers ordered by the outside pathologist, and then routinely stain for claudin-4 (if not already done) to definitively exclude an epithelial tumor.

\section{Caveats in lineage determination}

Certain pitfalls should be kept in mind when interpreting immunomarkers considered to indicate a specific site of origin for carcinoma. First, although PAX8 has conventionally been considered as a highly specific marker for müllerian, thyroid, and renal carcinoma in the differential diagnosis with mesothelioma (57), more recent data indicate that PAX8 is expressed in $6-18 \%$ of malignant peritoneal mesotheliomas (including strong diffuse expression in some cases), up to $60 \%$ of welldifferentiated papillary mesotheliomas, and up to one third of reactive mesothelial lesions in the peritoneum $(15,25,60,61)$. Mesothelial PAX8 expression appears to be somewhat more common in women, which may point to some biological overlap with müllerian tumors, although this remains unproven (60). Usefully, expression of estrogen receptor and progesterone receptor is exceptionally rare in peritoneal mesotheliomas $(0-2 \%$ and $0-7 \%$, respectively, in published studies) and thus provides strong evidence against a diagnosis of mesothelioma $(9,14,15,60,62)$.

The differential diagnosis between sarcomatoid carcinoma and sarcomatoid mesothelioma is notoriously difficult. Pan-cytokeratin immunohistochemistry is approximately $90 \%$ sensitive for both sarcomatoid mesothelioma and sarcomatoid carcinoma (34), but the sensitivity and specificity of other mesothelial and epithelial markers is poor in sarcomatoid tumors, as discussed above. Recent guidelines for diagnosis of sarcomatoid lesions provide practical guidance in these cases (34). A recent small study reported that nuclear GATA3 expression is $100 \%$ sensitive and $85 \%$ specific for sarcomatoid mesothelioma in the differential diagnosis with sarcomatoid carcinoma of the lung (63) (Figure 4). Similarly, MUC4 expression is reportedly $72 \%$ sensitive and $100 \%$ specific for diagnosis of sarcomatoid carcinoma of the lung, in the differential diagnosis with sarcomatoid mesothelioma (37). Both of these promising findings await validation in a larger, carefully designed multi-institutional study.

In the differential diagnosis with carcinoma of the lung or serous ovarian carcinoma, BAP1 loss is virtually $100 \%$ specific for mesothelioma $(15,50,60,64,65)$. Accordingly, in these specific differentials, BAP1 loss serves as a marker of both mesothelial lineage and malignancy (see also below). However, BAP1 loss is common in other tumor types, including renal cell carcinoma, and is therefore not intrinsically diagnostic of malignant mesothelioma in all circumstances $(66,67)$.

Rarely, the morphologic differential diagnosis for malignant mesothelioma includes non-epithelial tumors (including mesenchymal, hematologic, and melanocytic lesions, among others). In these rare cases, rote application of a panel of mesothelial and epithelial markers could prove confusing or frankly misleading. We reiterate that careful morphologic assessment remains the cornerstone of diagnosis, and should guide application of the appropriate diagnostic immunopanel $(4,34)$.

\section{Discriminating benign and malignant mesothelial lesions}

In tumors with overtly malignant morphologic features, immunohistochemical confirmation of mesothelial lineage may be sufficient for diagnosis of mesothelioma. In cases without overtly malignant morphology, however, additional immunostains can assist in distinction of benign and malignant mesothelial proliferations. This is of great help in small biopsies and cytology preparations, in which the tumor's relationship to surrounding tissues may be altogether absent $(65,68-73)$. None of these markers is without caveats, and interpretation in the context of morphology and other ancillary studies (see Figure 1) is imperative.

Numerous immunomarkers have been proposed to distinguish benign from malignant mesothelial proliferations, but few have stood the test of time. As noted in the introduction, specificity is paramount in markers of malignant mesothelioma, given the grave implications of this diagnosis. New immunomarkers with high specificity 

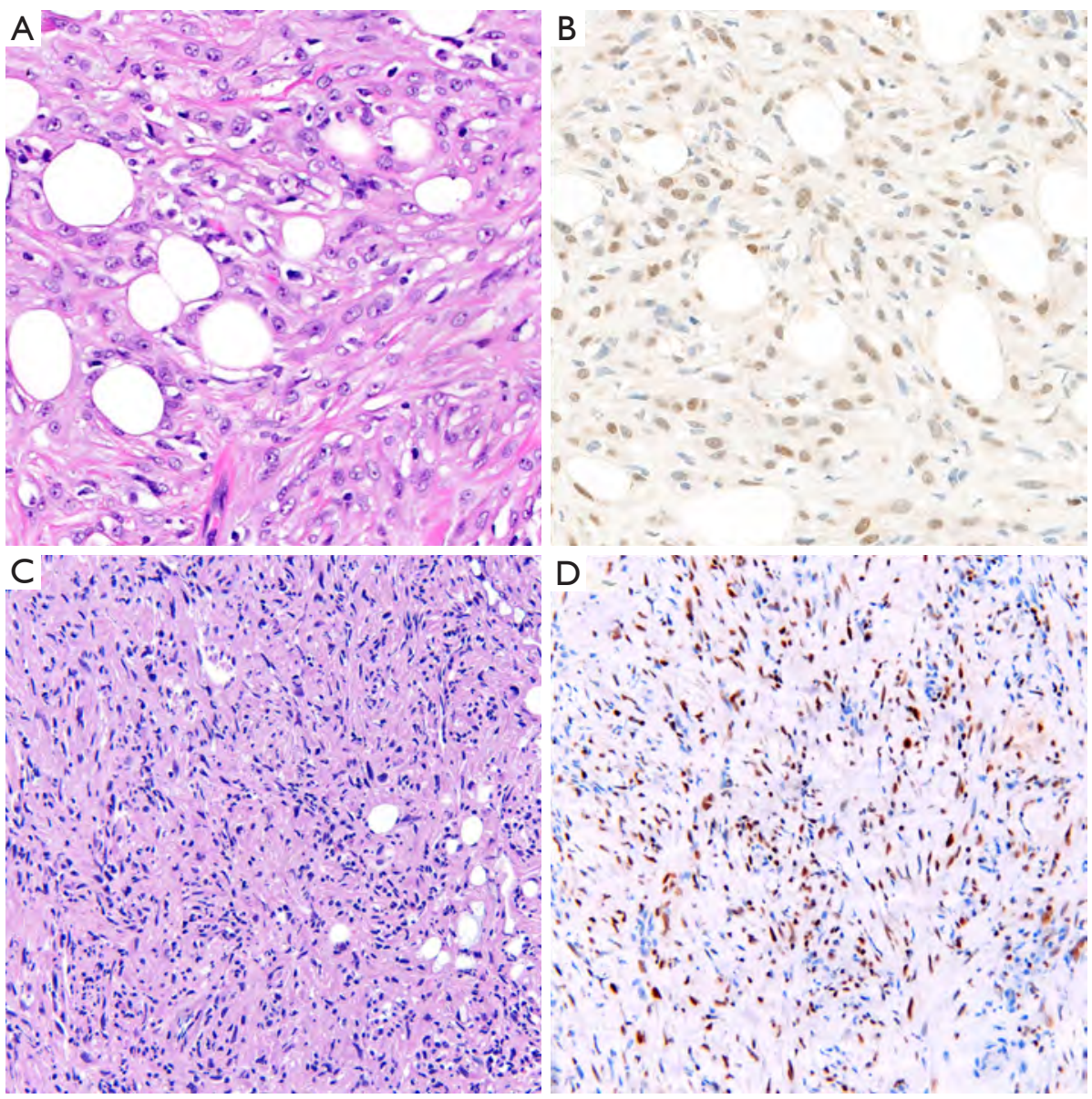

Figure 4 GATA3 expression is reportedly $72 \%$ sensitive and 100\% specific for diagnosis of malignant mesothelioma, in the differential with adenocarcinoma of the lung. GATA3 staining intensity may be weak, as in B. (A,C, hematoxylin and eosin; B,D, GATA3 immunostain; original magnification $\times 200$ ).

for malignancy in mesothelial lesions are therefore, quite appropriately, supplanting older, less specific markers in routine diagnostic practice. A recent publication provided a detailed review of five of these older mesothelioma markers, including desmin, EMA, GLUT-1, p53, and IMP-3 (74). The interested reader is referred there for further discussion of these markers, which we no longer recommend for routine diagnostic use. Reported sensitivity and specificity data for the discussed markers are summarized in Table 2.

\section{$B A P 1$}

Immunohistochemical loss of nuclear BRCA-associated protein 1 (BAP1) has emerged in recent years as a highly specific marker of malignancy in mesothelial lesions $(50,60,64,69-72,75-81)$. BAP1 loss in mesothelioma was originally described in the context of familial predisposition to mesothelioma in Cappadocia, Turkey, and the BAP1 germline mutation has since been recognized as the most common germline event predisposing to pleural and peritoneal mesothelioma, particularly in young patients with no history of radiation or asbestos exposure (82-84). $B A P 1$ alterations are also an important event in sporadic pleural and peritoneal mesothelioma $(75,85)$. In diagnostic immunohistochemistry, BAP1 is considered lost when tumor nuclear staining in absent in the presence of a positive internal control. Cytoplasmic-only staining (resulting from loss of BAP1's autodeubiquitination function) is seen in a subset of cases and is considered BAP1 loss for diagnostic purposes (86-88).

Loss of nuclear BAP1 staining by immunohistochemistry is highly concordant with BAP1 mutation $(75,86,89)$, 
Table 2 Sensitivity and specificity of malignant mesothelioma markers

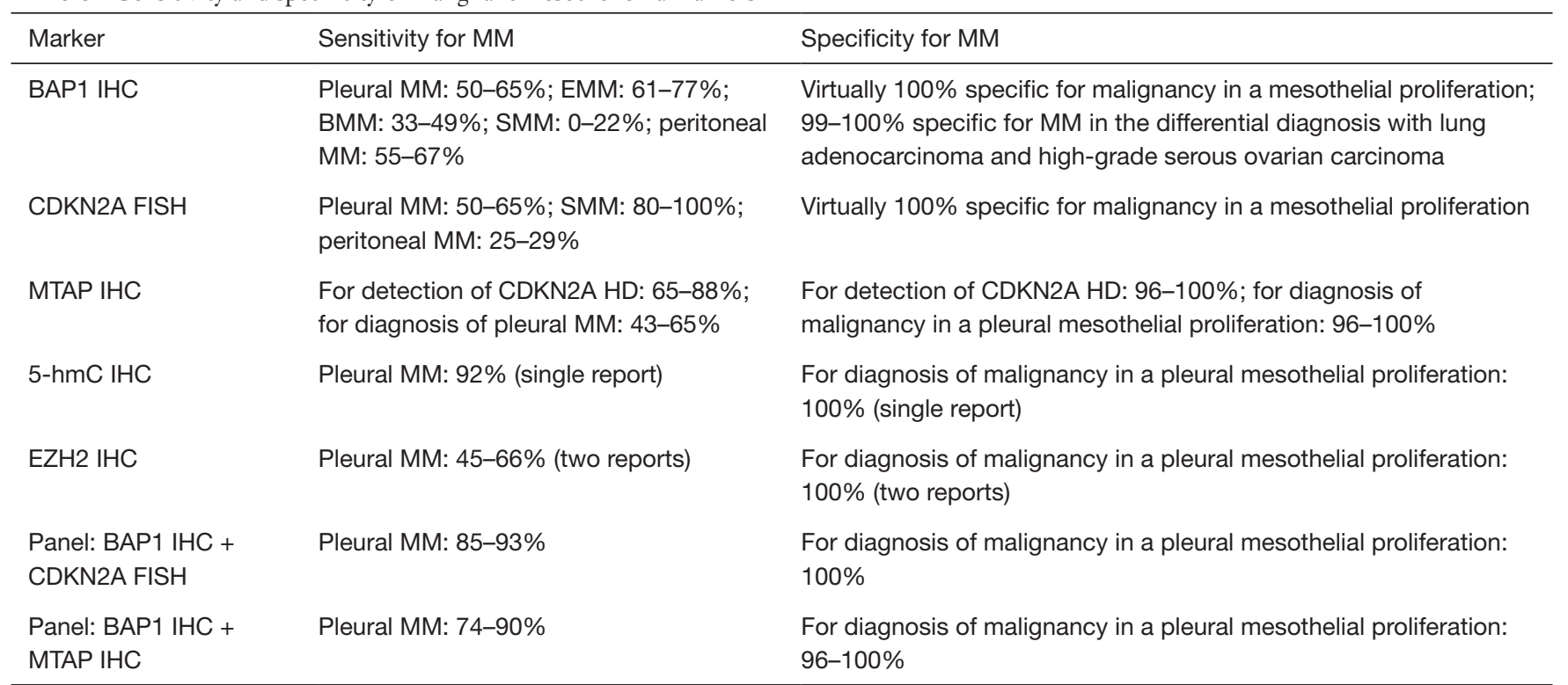

Except as noted for BAP1, specificity of these markers pertains to diagnosis of malignancy once mesothelial lineage has been established with lineage-specific markers. BMM, biphasic malignant mesothelioma; EMM, epithelioid malignant mesothelioma; FISH, fluorescent in situ hybridization; IHC, immunohistochemistry; MM, malignant mesothelioma; SMM, sarcomatoid malignant mesothelioma.

and BAP1 loss is virtually $100 \%$ specific for malignancy in mesothelial proliferations $(72,78,81,90-92)$ (Figure 5). Unfortunately, BAP1 loss is a somewhat insensitive marker, occurring in approximately $50-65 \%$ of all pleural mesotheliomas, including $61-77 \%$ of epithelioid, $33-49 \%$ of biphasic, and $0-22 \%$ of sarcomatoid mesotheliomas (70-72,75-80,86,89,90,92-96). Sensitivity of BAP1 loss for diagnosis of peritoneal mesothelioma has been reported at $55-67 \%(15,60,64,70,97)$.

The preponderance of evidence indicates that BAP1 immunohistochemistry can be accurately applied to cell block specimens $(70-72,90)$, although one study showed specificity of $86 \%$ for diagnosis of mesothelioma (98). Accordingly, caution is warranted in interpretation of BAP1 immunohistochemistry on cytology specimens, especially when rendering a primary diagnosis of malignant mesothelioma.

In the differential diagnosis with carcinoma of the lung, breast, and stomach, BAP1 loss is highly specific for malignant mesothelioma, with one study showing BAP1 loss in just $1 \%$ of lung cancers $(79,90,99,100)$. Multiple studies have also indicated that BAP1 loss is virtually $100 \%$ specific for mesothelioma in the differential diagnosis with ovarian carcinoma $(60,64,90)$. However, as noted above, multiple other malignancies (including renal cell carcinoma and melanoma) also frequently show BAP1 loss $(66,67,79)$. These tumors may occasionally metastasize to the serosal surfaces, so careful consideration of the clinical and radiographic data, tumor morphology, and lineage-specific markers is critical when interpreting BAP1 immunohistochemistry.

In addition to its diagnostic role, multiple studies indicate that BAP1 loss is a favorable prognostic marker in sporadic mesothelioma $(95,96,101,102)$, and mesotheliomas arising in the context of germline $B A P 1$ mutation have a relatively favorable prognosis (82). This is discussed in greater detail below.

\section{CDKN2A and MTAP}

In mesothelial proliferations without clear-cut morphology or BAP1 loss, fluorescence in situ hybridization (FISH) to detect homozygous deletion of $C D K N 2 A$ (which encodes p16 protein) remains the next best diagnostic study (see Figure 1). Like BAP1 loss, CDKN2A homozygous deletion is virtually $100 \%$ specific for malignancy in mesothelial proliferations, but only $48-88 \%$ sensitive for diagnosis of pleural mesothelioma, with most figures in the range of $50-65 \%(77,81,92,103-105)$, and up to $80-100 \%$ sensitivity for diagnosis of sarcomatoid mesothelioma, specifically 

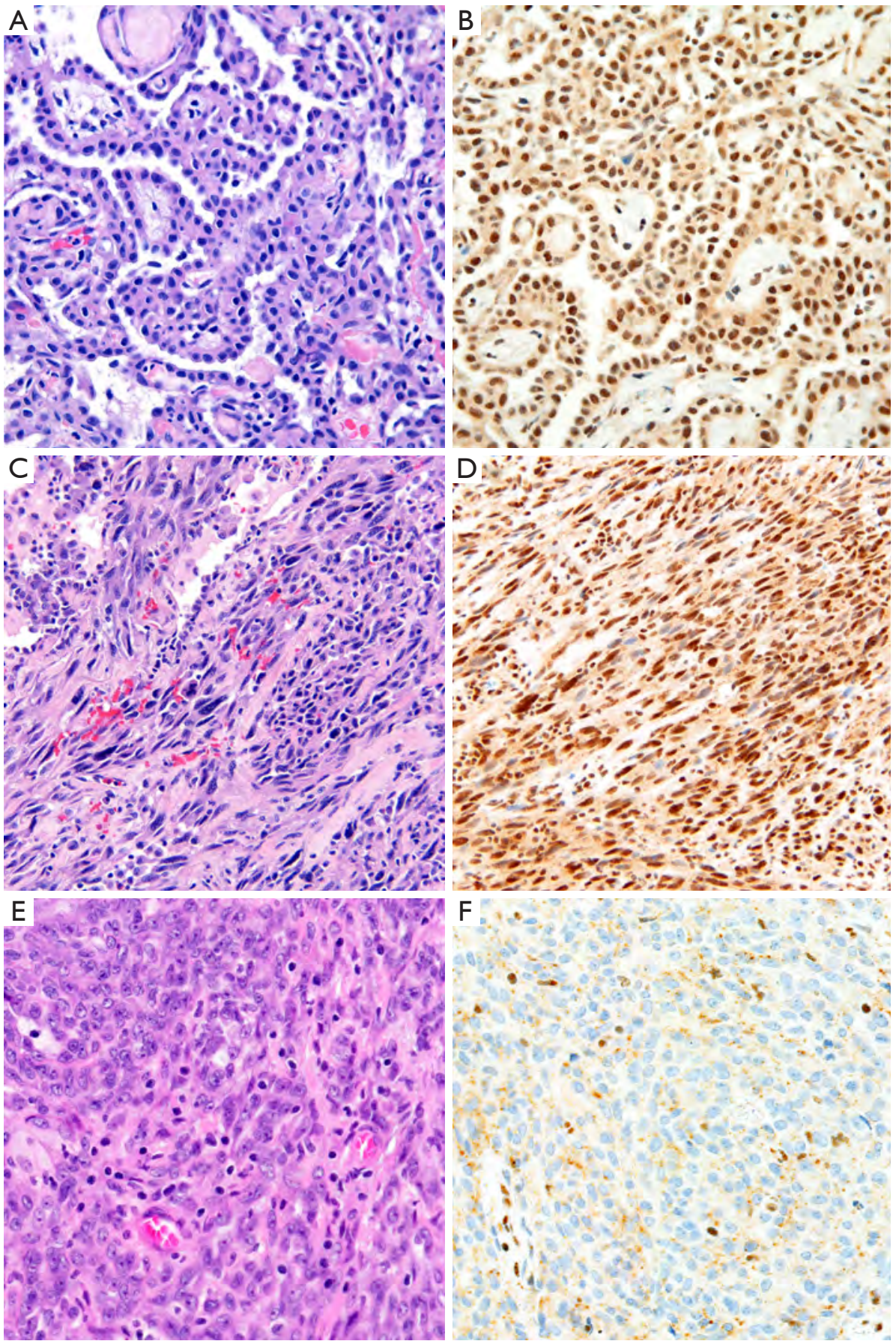

Figure 5 BAP1 loss is highly specific for malignancy in a mesothelial lesion. (A,B) BAP1 retention in papillary mesothelial hyperplasia (A, hematoxylin and eosin; B, BAP1 immunostain; original magnification $\times 400$ ). (C,D) BAP1 retention in a sarcomatoid malignant mesothelioma. BAP1 loss is seen in only some $15 \%$ of sarcomatoid mesothelioma. (E,F) BAP1 loss in an epithelioid malignant mesothelioma. Approximately 70\% of epithelioid malignant mesotheliomas show BAP1 loss. (C,E, hematoxylin and eosin; D,F, BAP1 immunostain; original magnification $\times 200)$. BAP1, BRCA-associated protein 1 . 
$(76,94,106)$. In the peritoneum (where sarcomatoid morphology is significantly rarer), sensitivity of $C D K N 2 A$ FISH is lower, at $25-29 \%(97,105)$.

Because immunohistochemistry is more widely available and less expensive than FISH, there has been interest in identifying an immunohistochemical surrogate for CDKN2A FISH; p16 immunohistochemistry, however, has proved unreliable (78). Nearly twenty years ago, cytogenetic studies revealed frequent co-deletion of the neighboring MTAP gene (encoding methylthioadenosine phosphorylase, involved in adenosine salvage) with $C D K N 2 A$ at the 9p21 locus (107-109), raising the potential for MTAP immunohistochemistry as a surrogate for $C D K N 2 A$ deletion. One early study of MTAP immunohistochemistry showed poor concordance with $C D K N 2 A$ FISH (110), but multiple more recent studies have shown that loss of cytoplasmic MTAP staining is $65-88 \%$ sensitive and $96-100 \%$ specific for $C D K N 2 A$ homozygous deletion, and $43-65 \%$ sensitive and up to $100 \%$ specific for diagnosis of mesothelioma $(72,73,78,91,92,111)$, including in sarcomatoid mesothelioma (112) (Figure 6). The literature currently contains only one example of a mesothelioma with MTAP loss by immunohistochemistry but normal $C D K N 2 A$ copy number by FISH (111), and no reports of MTAP loss in a benign mesothelial lesion. MTAP immunohistochemistry is relatively easy to interpret, shows excellent interobserver reproducibility (111), and has been validated for application to cell block specimens $(72,73)$. Although the published case of MTAP loss in a tumor with normal $C D K N 2 A$ copy number raises some concern about specificity, we have begun using MTAP immunohistochemistry in routine clinical diagnosis, in close correlation with histomorphology and BAP1 immunohistochemistry, and reserving the use of confirmatory $C D K N 2 A$ FISH for challenging cases.

Thorough validation of these highly specific markers of malignant mesothelioma has led to the introduction of diagnostic panels into routine practice, combining different markers to enhance sensitivity. A diagnostic panel including both BAP1 immunohistochemistry and $C D K N 2 A$ FISH achieves a sensitivity of $58-93 \%$, with most reported figures in the $85-93 \%$ range $(72,77,78,81,92,94)$. By comparison, an immunopanel of BAP1 and MTAP is $74-90 \%$ sensitive for diagnosis of malignant mesothelioma $(72,78,91,92)$, in keeping with the imperfect sensitivity of MTAP loss for $C D K N 2 A$ deletion.

Importantly, the available literature on MTAP immunohistochemistry for diagnosis of mesothelioma pertains only to mesothelial proliferations of the pleura. There are no published data on MTAP immunohistochemistry in peritoneal mesothelial lesions, although we anticipate that the number of such lesions showing MTAP loss would be low, as $C D K N 2 A$ homozygous deletion is a relatively rare phenomenon in peritoneal mesothelioma. Indeed, in a cohort of 33 peritoneal mesotheliomas subjected to MTAP immunohistochemistry in our lab, only 1 showed MTAP loss (unpublished data). It should also be emphasized that $C D K N 2 A$ homozygous deletion is seen in a broad spectrum of malignant tumors, and that $C D K N 2 A$ deletion (or, by extension, MTAP loss) warrants a diagnosis of malignant mesothelioma only after mesothelial lineage has been firmly established.

\section{5-bydroxymethylcytosine (5-bmC)}

$5-\mathrm{hmC}$ is a modified nucleotide, produced from 5 -methylcytosine by the TET family of DNA hydroxylases as the first step of DNA demethylation (113-116). Discovery of 5 -hmC coincided with the larger realization that DNA methylation is, in fact, a reversible epigenetic phenomenon. Decreased levels of 5 -hmC have been reported in a broad range of malignancies, including in seminal studies of acute myeloid leukemia, glioblastoma, and melanoma $(117,118)$. There have been few reports on the significance of 5 -hmC in mesothelioma. Early work using rat and human cell lines implicated decreased TET expression and decreased global 5 -hmC in mesothelioma tumorigenesis (119). In a study of 49 mesotheliomas and 23 reactive mesothelial proliferations, our group found that loss of 5 -hmC staining in greater than $50 \%$ of tumor nuclei was $100 \%$ specific and $92 \%$ sensitive for diagnosis of malignancy (120) (Figure 7). These promising results await validation in a larger multi-institutional study, using more recently available monoclonal antibodies. Application of 5 -hmC immunohistochemistry has not yet been studied in small biopsies or cytology cell blocks, where quantification of tumor 5-hmC loss may present additional challenges.

As with MTAP immunohistochemistry, data on $5-\mathrm{hmC}$ immunohistochemistry have only been reported for mesothelial lesions of the pleura (120). Of 89 malignant peritoneal mesotheliomas stained in our laboratory for 5 -hmC, only $52(58 \%)$ showed loss of 5 -hmC in greater than $50 \%$ of tumor nuclei (unpublished data), suggesting that sensitivity of 5-hmC immunohistochemistry for diagnosis of peritoneal mesothelioma may be lower than for pleural mesothelioma.

Molecular assays for 5 -hmC have been developed 


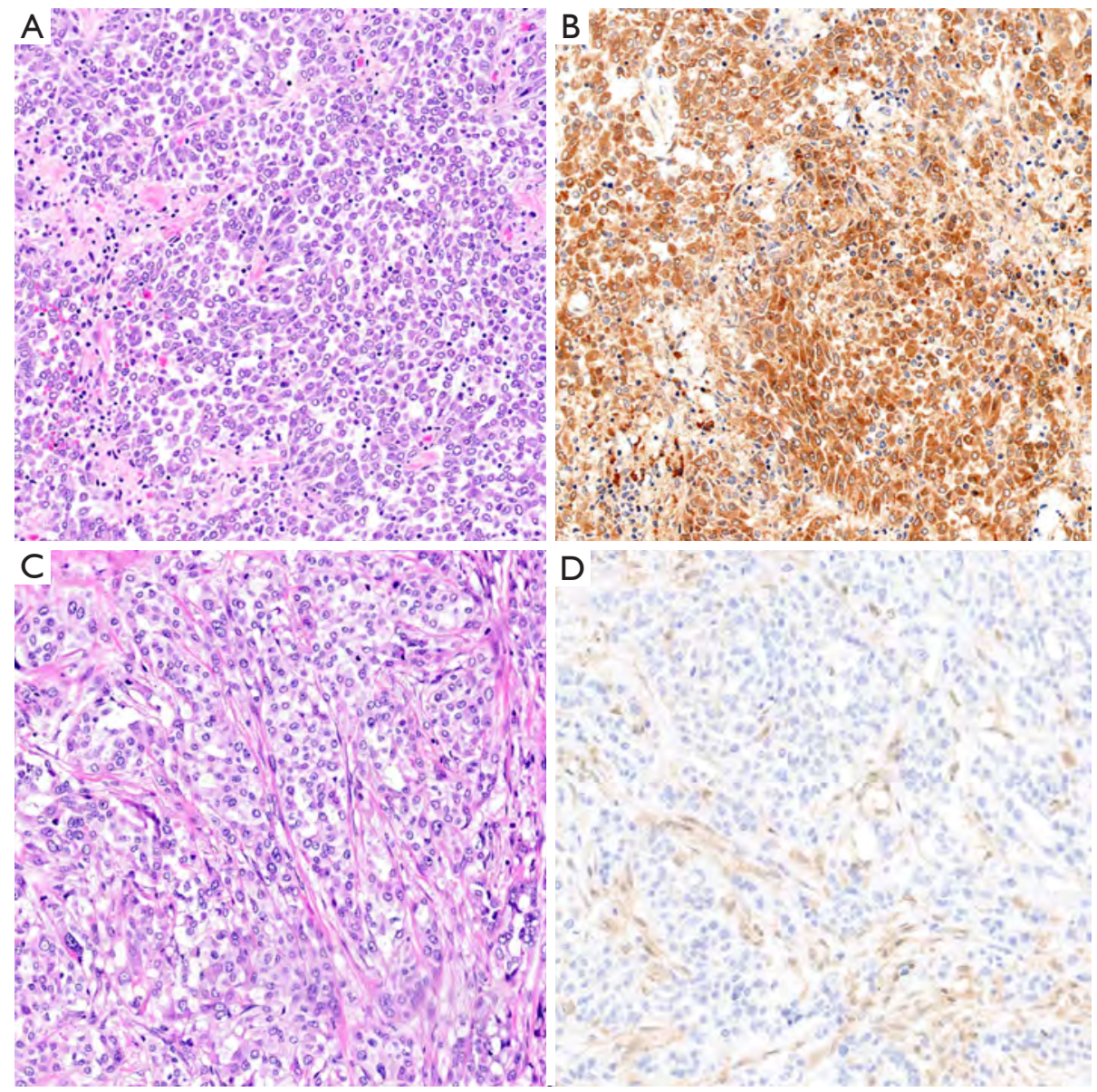

Figure 6 MTAP loss is a surrogate for CDKN2A homozygous deletion and is highly specific for malignancy in a mesothelial lesion. (A,B) MTAP retention in an epithelioid malignant mesothelioma. (A, hematoxylin and eosin; B, MTAP immunostain; original magnification $\times 100)$. (C,D) MTAP loss in an epithelioid malignant mesothelioma. MTAP loss is approximately $80 \%$ sensitive for detecting CDKN2A homozygous deletion and $60 \%$ sensitive for diagnosis of malignant mesothelioma. (C, hematoxylin and eosin; D, MTAP immunostain; original magnification $\times 100$ ). MTAP, methylthioadenosine phosphorylase.

recently, and provide substantially more detailed information on 5-hydroxymethylation patterns than can be achieved with immunohistochemistry. Molecular studies investigating the tumor-specific 5 -hmC profile in mesothelioma are underway.

\section{EZH2}

Enhancer of zeste homolog 2 (EZH2) is a component of polycomb repressive complex 2 (PRC2) and plays a central role in epigenetic suppression of gene expression through trimethylation of a critical lysine residue in histone 3 (H3K27) (121). Overexpression of EZH2 is reported in a range of malignancies, and a link between
BAP1 loss and overexpression of EZH2 suggests a role for the PRC2 pathway and H3K27 hypermethylation in mesothelioma development and progression (122). Early work demonstrated significantly higher EZH2 expression in malignant mesothelioma compared to reactive mesothelial lesions, whereas knockdown of EZH2 inhibits mesothelioma tumorigenesis $(5,123)$. In two recent studies of mesothelial proliferations, expression of EZH2 in $>50 \%$ of lesional cells was found to be $45-66 \%$ sensitive and $100 \%$ specific for malignant mesothelioma, with sensitivity increasing to $74-90 \%$ for an immunopanel including BAP1 and EZH2 $(80,92)$.

Importantly, as with loss of MTAP and 5-hmC, overexpression of $\mathrm{EZH} 2$ is not specific for mesothelioma in 


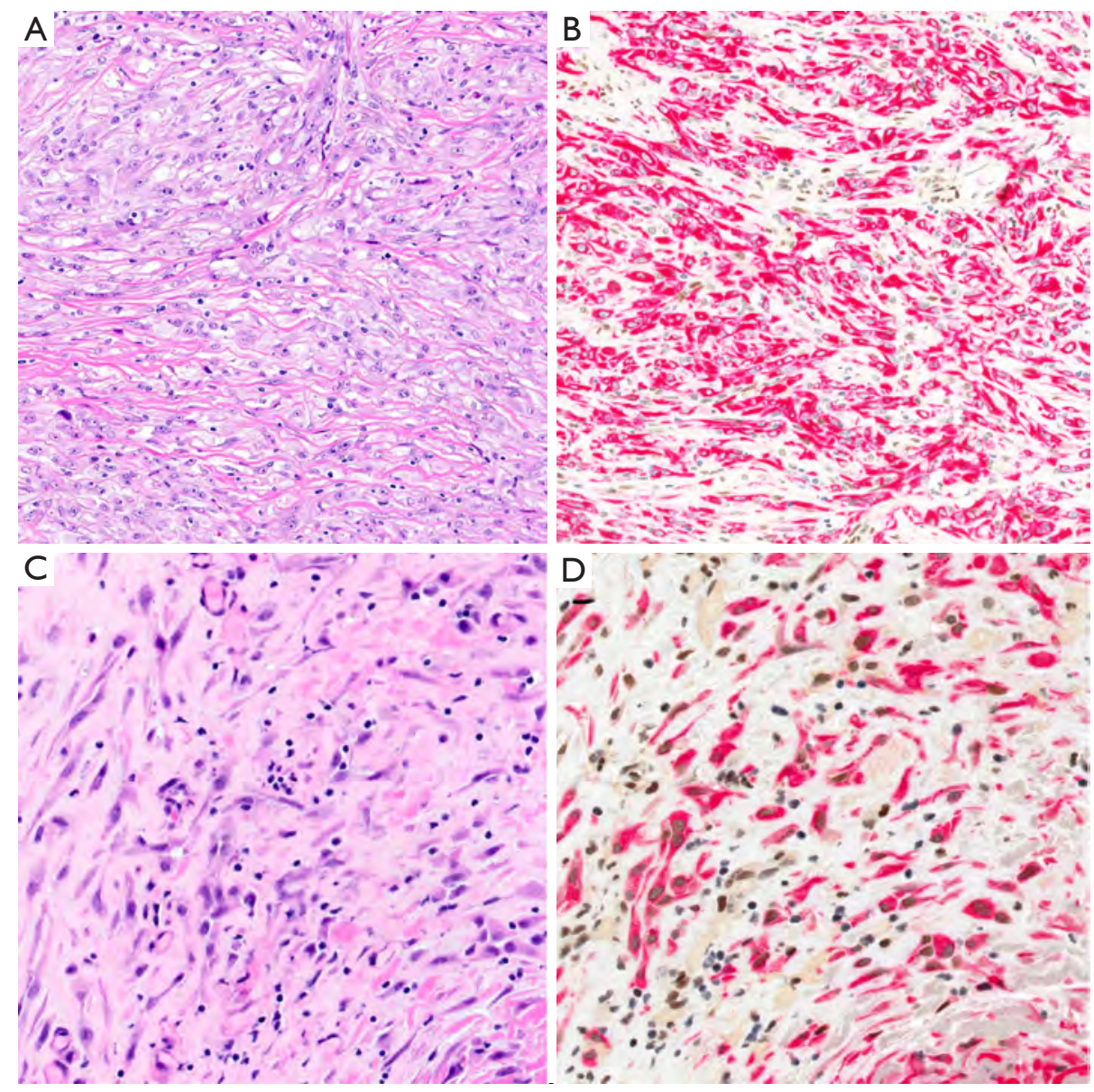

Figure 7 Loss of nuclear 5-hydroxymethylcytosine (5-hmC) staining is sensitive and specific for malignancy in a mesothelial lesion. (A,B) Extensive 5-hmC loss in a sarcomatoid malignant mesothelioma [A, hematoxylin and eosin; B, CAM5.2/5-hmC double immunostain (CAM5.2 in red, 5-hmC in brown); original magnification $\times 100]$. (C,D) Nuclear 5-hmC is largely retained in this reactive spindled mesothelial proliferation [C, hematoxylin and eosin; D, CAM5.2/5-hmC double immunostain (CAM5.2 in red, 5-hmC in brown); original magnification $\times 200]$.

the differential diagnosis with other malignant tumors (124), and should only be used to support this diagnosis once mesothelial lineage has been confidently established. Although EZH2 is a promising marker of malignant mesothelioma, we are awaiting validation in larger multiinstitutional studies before adopting this marker in our routine diagnostic practice.

\section{Use of immunomarkers to subtype malignant mesothelioma}

Once a diagnosis of malignant mesothelioma is made, the tumor should be designated as epithelioid, biphasic, or sarcomatoid histotype. This classification has significant prognostic and therapeutic implications, but not all lesions can be easily classified morphologically. A recent study found only moderate agreement among pathologists with mesothelioma expertise in assignment of biphasic histotype (125). Here we discuss two problematic areas where immunohistochemistry may be useful. First, cytokeratin immunostaining may help to distinguish sarcomatoid mesothelioma from epithelioid mesothelioma in challenging cases. Second, application of malignancy-specific markers may help distinguish biphasic mesothelioma from epithelioid mesothelioma associated with reactive spindled mesothelial cells.

In challenging cases, a cytokeratin immunostain may 
assist in distinguishing sarcomatoid and epithelioid patterns by highlighting greater cell-cell cohesion in the latter (Figure 8). This approach is sometimes used as an adjunct in diagnosis of biphasic mesothelioma. In the recent study of interobserver agreement in diagnosis of biphasic mesothelioma referenced above, participating pathologists reported that a cytokeratin immunostain aided histotype classification in $77 \%$ of cases (125). However, there are no objective criteria for interpreting cytokeratin immunostains in this context, and the diagnosis is ultimately a subjective evaluation by the pathologist. In future, this application may also help delineate foci of transitional mesothelioma, although the immunohistochemical characteristics of this provisional morphologic subtype are not yet fully elucidated.

Use of immunohistochemistry to distinguish reactive from malignant spindled cells-and thus to distinguish true biphasic mesothelioma from epithelioid mesothelioma with adjacent reactive spindled mesothelial cellsremains an area of controversy. In one study of 43 biphasic mesotheliomas, all 21 tumors with BAP1 loss showed concordant loss in both the epithelioid and sarcomatoid components (95). A subsequent study found BAP1 loss in 5 of 13 biphasic mesotheliomas, with concordant BAP1 staining between the epithelioid and sarcomatoid elements in all 13 cases (76). These studies suggest that, when associated with an epithelioid mesothelioma with BAP1 loss, spindled mesothelial cells with intact BAP1 are likely reactive. In contrast, however, a recent study of 42 biphasic mesotheliomas found that only 11 of 21 cases with BAP1 loss showed concordant loss in both tumor elements (125) (Schulte, unpublished data). Similarly, in one recent study of 13 tumors diagnosed as biphasic mesothelioma on the basis of morphology, the authors suggested that 5 tumors be reclassified as epithelioid mesothelioma on the basis of BAP1 loss in the epithelioid component and BAP1 retention in the sarcomatoid component (86). Considering these studies together, it seems that their differing conclusions likely stem, at least in part, from lack of an objective gold standard method for definitively classifying challenging cases as epithelioid or biphasic, thus introducing the potential for circular reasoning. In our practice, we presently regard BAP1 loss in spindled mesothelial cells as specific but not sensitive for confirming a diagnosis of biphasic mesothelioma. That is, retention of BAP1 in a spindled mesothelial population does not, in this context, make it benign.

$$
\text { Analogously, } C D K N 2 A \text { FISH or MTAP }
$$

immunohistochemistry could also be used to distinguish true biphasic mesothelioma from epithelioid mesothelioma with an associated reactive spindle component. In fact, one might intuit that this approach would be more sensitive than BAP1 immunohistochemistry, given the higher incidence of $C D K N 2 A$ homozygous deletion in sarcomatoid mesothelioma. $\mathrm{Wu}$ and colleagues found that the epithelioid and sarcomatoid components of a biphasic mesothelioma show concordant $C D K N 2 A$ status by FISH in 29 of 29 cases (76). This suggests that $C D K N 2 A$ FISH could be both sensitive and specific for confirming a diagnosis of biphasic mesothelioma in tumors with $C D K N 2 A$ homozygous deletion in the epithelioid element, although this may prove burdensome, if FISH must be performed twice to capture both tumor elements. MTAP immunohistochemistry, as a surrogate for $C D K N 2 A$ FISH, could be a more practical approach, but this application has not yet been formally studied.

Our group also found that extensive $5-\mathrm{hmC}$ loss in a spindled mesothelial proliferation is characteristic of malignancy and may warrant diagnosis of biphasic mesothelioma when located adjacent to a malignant epithelioid component (120) (Figure 9). New data suggest that molecular-based epigenetic profiling can accurately identify mesothelioma histotype (unpublished data, presented USCAP 2019, National Harbor, MD, USA), although this technique is not yet in clinical use.

\section{Prognostic and predictive markers}

Beyond their role in mesothelioma diagnosis, immunomarkers can also provide clinically valuable prognostic and predictive information. Although numerous markers are reportedly related to prognosis in mesothelioma, we will highlight only those markers currently used in routine diagnostic practice.

The preponderance of prognostic studies supports a better outcome in mesothelioma with BAP1 loss $(80,89,93,95,96,101,102,126)$, although one study found that BAP1 loss portends a poorer prognosis and suggests that reports of improved prognosis may reflect confounding (127). Published data on BAP1 loss in peritoneal mesothelioma also suggest improved survival (85), although some have found no significant prognostic effect (97). Importantly, these studies have not generally discriminated between germline and sporadic BAP1 loss.

Studies specific to BAP1 loss secondary to germline $B A P 1$ mutation have found a significantly better prognosis 

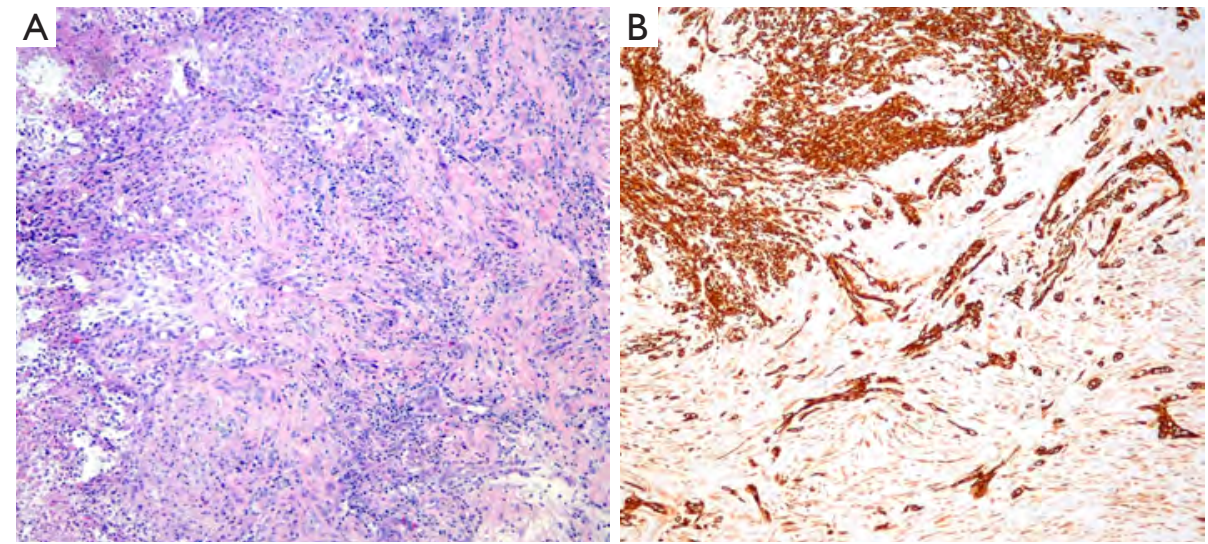

Figure 8 A cytokeratin immunostain on a biphasic malignant mesothelioma contrasts cohesive epithelioid growth (upper left) from spindled single-cell sarcomatoid growth (lower right) (A, hematoxylin and eosin; B, CAM5.2 immunostain; original magnification $\times 100)$.

compared to non-germline-mutated mesothelioma, irrespective of tumor site, with 5 -year overall survival of $47 \%$, and $7 \%$, respectively $(82,128)$. Among patients with $B A P 1$ germline mutation, peritoneal mesothelioma is associated with significantly longer overall survival than pleural mesothelioma (median 10 and 2 years, respectively, versus 9 months for patients with non-germline-mutated pleural or peritoneal mesothelioma) (82). Although improved outcomes in mesothelioma with germline BAP1 mutation may be partly attributable to active surveillance and earlier detection going forward, better survival in these patients predates the discovery of germline BAP1 syndrome, suggesting that differences in tumor biology also play a role (82). We are still awaiting a single unifying study, comparing outcomes in mesotheliomas with retained BAP1, sporadic BAP1 loss, and germline BAP1 mutation, which may permit further prognostic stratification of patients.

Multiple studies have shown that homozygous deletion of $C D K N 2 A$ is associated with shorter survival in both pleural and peritoneal mesothelioma $(97,101,109,129,130)$. An analogous prognostic function for MTAP immunohistochemistry is logical, but has not yet been validated. The prognostic role of EZH2 immunohistochemistry has been examined in only a limited capacity, with one study showing poorer outcomes in patients with high EZH2 expression (92) and a second study showing no association between EZH2 expression and outcome (80). Together, these two studies had only 80 patients, and larger studies are needed to establish the prognostic significance of EZH2 expression.

Programmed death-ligand 1 (PD-L1) expression in mesothelioma has been associated with shorter survival, compared to mesotheliomas without PD-L1 expression (131-137). Interaction between PD-L1 on tumor cells and PD-1 on tumor infiltrating lymphocytes downregulates the anti-tumor immune response, and therapeutic targeting of this interaction represents a major advance in clinical oncology. Positive PD-L1 expression ( $>1 \%$ tumor cell staining) is reported in $11-72 \%$ of mesotheliomas, with substantial variation between different laboratories and different anti-PD-L1 antibody clones (131-138). Pembrolizumab (an anti-PD-1 monoclonal antibody) is now accepted as salvage therapy for mesothelioma with PD-L1 expression by immunohistochemistry, and nivolumab (another anti-PD-1 agent) is accepted as salvage therapy regardless of PD-L1 immunohistochemistry findings. This highlights the uncertain value of $\mathrm{PD}$ L1 immunohistochemistry as a predictive marker for immunotherapy response, with conflicting results for different agents in a rapidly evolving therapeutic landscape. Notwithstanding, we perform PD-L1 immunostaining on all mesotheliomas at our institution, and PD-L1 immunohistochemistry is the subject of multiple ongoing clinical trials. The interested reader is referred to a recent excellent review for an in-depth discussion from the clinical perspective (139).

\section{Rare sites and unusual tumors}

\section{Mesothelioma in situ}

The existence of an in situ precursor to mesothelioma has long been postulated, but the concept had previously 

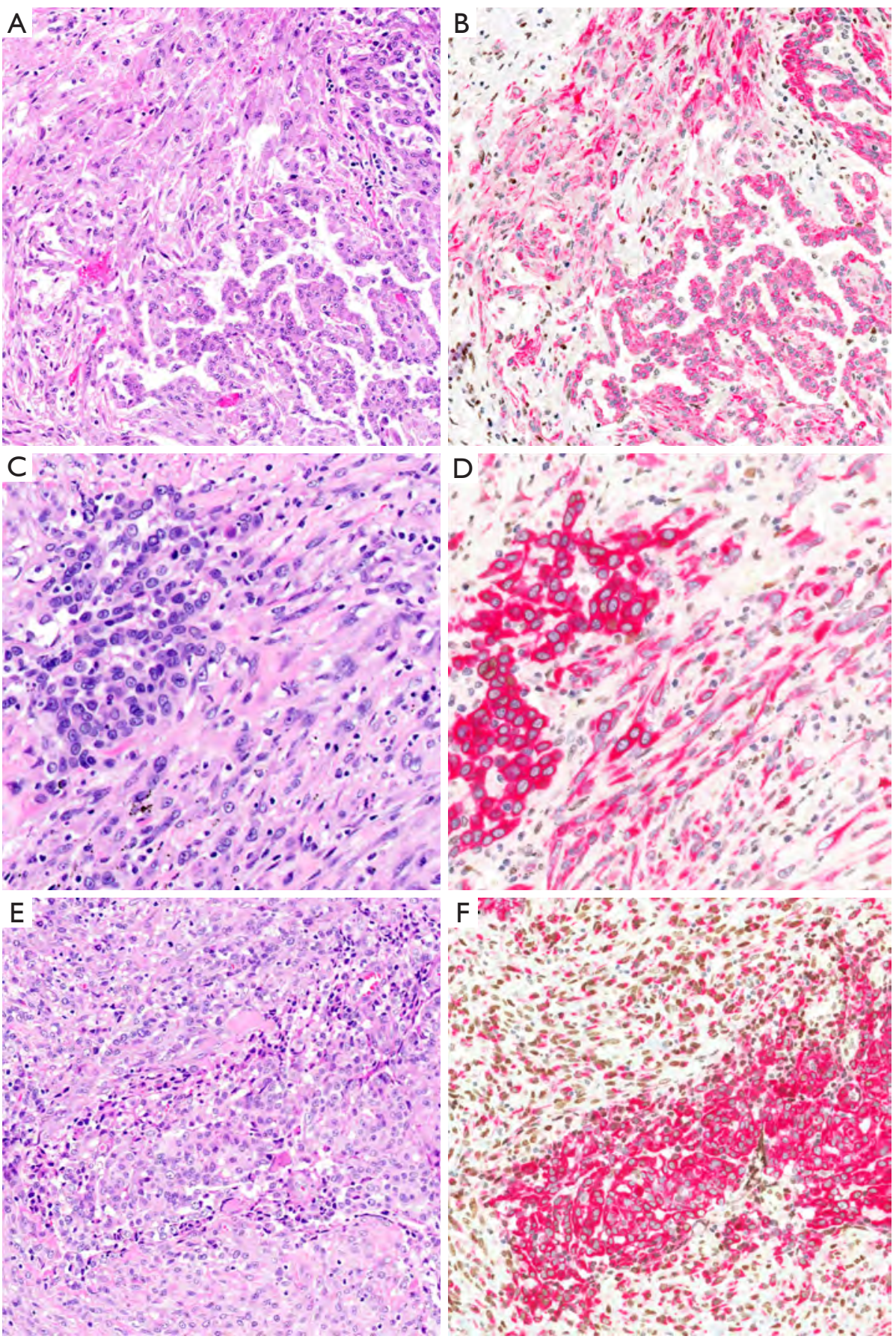

Figure 9 Immunohistochemistry for 5-hydroxymethylcytosine may aid in mesothelioma subtyping. (A,B) In true biphasic malignant mesothelioma, both the epithelioid and sarcomatoid elements show extensive loss of 5-hydroxymethylcytosine (5-hmC) [A, hematoxylin and eosin; B, CAM5.2/5-hmC double immunostain (CAM5.2 in red, 5-hmC in brown); original magnification $\times 100]$. (C,D) Biphasic malignant mesothelioma with extensive loss of 5-hmC in both epithelioid and sarcomatoid tumor elements [C, hematoxylin and eosin; D, CAM5.2/5hmC double immunostain (CAM5.2 in red, 5-hmC in brown); original magnification $\times 200]$. (E,F) An epithelioid malignant mesothelioma with an associated reactive spindled mesothelial population. The epithelioid mesothelioma shows extensive 5 -hmC loss, whereas the adjacent reactive spindled component shows extensive nuclear 5-hmC staining [E, hematoxylin and eosin; F, CAM5.2/5-hmC double immunostain (CAM5.2 in red, 5-hmC in brown); original magnification $\times 100$ ]. 
gained little credibility, due to difficulties both in definition and in detection $(140,141)$. Two recent related series characterizing a total of 12 cases of apparent mesothelioma in situ (11 pleural, 1 peritoneal) have prompted a renewed interest in the concept $(142,143)$. Churg and colleagues propose a narrow and rigorous definition of malignant mesothelioma in situ: (I) a single layer of surface mesothelial cells, (II) immunohistochemical loss of BAP1, and (III) no radiographic or clinical evidence of a tumor. (To improve specificity, their studies also excluded any patients who developed invasive mesothelioma within 1 year of the in situ diagnosis). Note that ancillary diagnostic studies (BAP1 loss or, in principle, MTAP loss or $C D K N 2 A$ homozygous deletion) are necessary to diagnose mesothelioma in situ, because its constituent cells overlap cytologically with reactive mesothelium, The precise significance of incidentally discovered mesothelioma in situ is unclear, although the recent series reported progression to mesothelioma in 7 of 10 patients, over the course of $1-8$ years (143). Given the clinical and diagnostic uncertainty that continues to surround malignant mesothelioma in situ, we would recommend that this diagnosis be made only in consultation with a pathologist with expertise in mesothelioma.

\section{Localized malignant mesothelioma}

Localized malignant mesothelioma is a rare malignant neoplasm that may arise in the pleura or peritoneum and accounts for approximately $1 \%$ of all malignant mesothelioma diagnoses. In contrast to more typical diffuse malignant mesothelioma, localized malignant mesothelioma is defined by sharp circumscription and relatively good prognosis, with reported median survival ranging from 29134 months in three studies (144,145) (Marchevsky et al., in press, Modern Pathology, 2019). Despite these clinical differences, the histomorphology and immunophenotype of localized and diffuse mesothelioma are identical, including the same relative frequency of epithelioid, biphasic, and sarcomatoid histotype in localized and diffuse tumors. Localized mesothelioma is also associated with asbestos exposure in approximately one-third of cases. Although studies of localized malignant mesothelioma are limited, recent data by Marchevsky and colleagues (in press, Modern Patbology, 2019) indicate that the ancillary studies discussed in this review, including immunohistochemical loss of BAP1, can be applied to diagnosis of localized malignant mesothelioma. These studies may be particularly useful in this setting, as localized malignant mesothelioma is a rare tumor that can closely mimic non-mesothelial tumors and benign localized mesothelial proliferations. For a thorough and current review of available data on localized mesothelioma, the interested reader is referred to the above-cited forthcoming manuscript by Marchevsky and colleagues.

\section{Tumors at rare sites: paratesticular and pericardial mesothelioma}

Paratesticular mesothelioma is an exceptionally rare tumor, accounting for $<1 \%$ of mesothelioma diagnoses in men. Because it arises from the tunica vaginalis, paratesticular mesothelioma would seem developmentally related to peritoneal mesothelioma. However, three published series, with a total of 49 cases, point to morphological and clinical differences (146-148). Some studies suggest that biphasic morphology may be more common in mesothelioma of the tunica vaginalis $(146,147)$, and clinical outcomes are poor. In one study of 6 patients, 5 had died of disease over 8-74 months (146). Although immunohistochemical testing was limited in two studies by retrospective review, mesothelial and epithelial markers appear to show the same sensitivity and specificity for paratesticular mesothelioma as at other sites (146-148). Markers of mesothelial malignancy have not yet been studied in paratesticular mesothelioma, to our knowledge.

Pericardial mesothelioma is similarly rare, comprising $<1 \%$ of all mesothelioma diagnoses. Pericardial mesothelioma appears to be clinically, morphologically, and immunophenotypically similar to pleural mesothelioma. In a recent large series of 61 pericardial mesotheliomas (reported to date in abstract form only), $82 \%$ of tumors were epithelioid, $13 \%$ biphasic, and 5\% sarcomatoid, and clinical outcomes were poor, with median survival of 13 months and only $11 \% 24$-month survival. Immunohistochemistry for cytokeratin AE1/AE3, calretinin, CK5/6, and WT-1 showed sensitivity of $100 \%, 94 \%, 90 \%$, and $81 \%$, respectively (149). We are awaiting publication of a full manuscript for details on application of malignancy-specific immunomarkers to pericardial mesothelioma, but we anticipate that they will be as useful in this setting as in pleural mesothelioma.

\section{Conclusions}

Immunohistochemistry plays an ever-larger role in accurate diagnosis of mesothelial lesions. Although mesothelial- 
and epithelial-specific markers have long been used to confirm mesothelial lineage, novel markers, such as claudin- 4 , continue to increase the accuracy of this determination. Multiple markers of malignancy (including immunohistochemistry for BAP1 and, more recently, MTAP) have entered routine diagnostic use in the last decade, and promising new markers are the subject of ongoing study. Beyond their diagnostic use, immunomarkers are also assuming a novel prognostic and predictive role, which will likely grow as targeted therapies are increasingly deployed in treatment of malignant mesothelioma.

\section{Acknowledgments}

Funding: The authors would like to thank Terri Li and University of Chicago Human Tissue Resource Center for their ongoing support of our mesothelioma research.

\section{Footnote}

Conflicts of Interest: The authors have no conflicts of interest to declare.

Ethical Statement: The authors are accountable for all aspects of the work in ensuring that questions related to the accuracy or integrity of any part of the work are appropriately investigated and resolved.

Open Access Statement: This is an Open Access article distributed in accordance with the Creative Commons Attribution-NonCommercial-NoDerivs 4.0 International License (CC BY-NC-ND 4.0), which permits the noncommercial replication and distribution of the article with the strict proviso that no changes or edits are made and the original work is properly cited (including links to both the formal publication through the relevant DOI and the license). See: https://creativecommons.org/licenses/by-nc$\mathrm{nd} / 4.0 \%$.

\section{References}

1. Beebe-Dimmer JL, Fryzek JP, Yee CL, et al. Mesothelioma in the United States: a Surveillance, Epidemiology, and End Results (SEER)-Medicare investigation of treatment patterns and overall survival. Clin Epidemiol 2016;8:743-50.

2. Rosen LE, Karrison T, Ananthanarayanan V, et al. Nuclear grade and necrosis predict prognosis in malignant epithelioid pleural mesothelioma: a multi-institutional study. Mod Pathol 2018;31:598-606.

3. Mazurek JM, Syamlal G, Wood JM, et al. Malignant Mesothelioma Mortality - United States, 1999-2015. MMWR Morb Mortal Wkly Rep 2017;66:214-8.

4. Husain AN, Colby TV, Ordonez NG, et al. Guidelines for Pathologic Diagnosis of Malignant Mesothelioma 2017 Update of the Consensus Statement From the International Mesothelioma Interest Group. Arch Pathol Lab Med 2018;142:89-108.

5. Chapel DB, Churg A, Santoni-Rugiu E, et al. Molecular pathways and diagnosis in malignant mesothelioma: A review of the 14th International Conference of the International Mesothelioma Interest Group. Lung Cancer 2019;127:69-75.

6. Ordóñez NG. Application of immunohistochemistry in the diagnosis of epithelioid mesothelioma: a review and update. Hum Pathol 2013;44:1-19.

7. Ordóñez NG. The immunohistochemical diagnosis of mesothelioma: a comparative study of epithelioid mesothelioma and lung adenocarcinoma. Am J Surg Pathol 2003;27:1031-51.

8. Ordóñez NG. The diagnostic utility of immunohistochemistry in distinguishing between epithelioid mesotheliomas and squamous carcinomas of the lung: a comparative study. Mod Pathol 2006;19:417-28.

9. Ordóñez NG. Value of immunohistochemistry in distinguishing peritoneal mesothelioma from serous carcinoma of the ovary and peritoneum: a review and update. Adv Anat Pathol 2006;13:16-25.

10. Ordóñez NG. Value of calretinin immunostaining in differentiating epithelial mesothelioma from lung adenocarcinoma. Mod Pathol 1998;11:929-33.

11. Ordóñez NG. The diagnostic utility of immunohistochemistry in distinguishing between mesothelioma and renal cell carcinoma: a comparative study. Hum Pathol 2004;35:697-710.

12. Granville LA, Younes M, Churg A, et al. Comparison of monoclonal versus polyclonal calretinin antibodies for immunohistochemical diagnosis of malignant mesothelioma. Appl Immunohistochem Mol Morphol 2005;13:75-9.

13. Hinterberger M, Reineke T, Storz M, et al. D2-40 and calretinin - a tissue microarray analysis of 341 malignant mesotheliomas with emphasis on sarcomatoid differentiation. Mod Pathol 2007;20:248-55.

14. Kawai T, Tominaga S, Hiroi S, et al. Peritoneal malignant mesothelioma (PMM), and primary peritoneal serous carcinoma (PPSC) and reactive mesothelial hyperplasia 
$(\mathrm{RMH})$ of the peritoneum. Immunohistochemical and fluorescence in situ hybridisation (FISH) analyses. J Clin Pathol 2016;69:706-12.

15. Tandon RT, Jimenez-Cortez Y, Taub R, et al. Immunohistochemistry in Peritoneal Mesothelioma: A Single-Center Experience of 244 Cases. Arch Pathol Lab Med 2018;142:236-42.

16. Kushitani K, Amatya VJ, Okada Y, et al. Utility and pitfalls of immunohistochemistry in the differential diagnosis between epithelioid mesothelioma and poorly differentiated lung squamous cell carcinoma. Histopathology 2017;70:375-84.

17. Kushitani K, Takeshima Y, Amatya VJ, et al. Immunohistochemical marker panels for distinguishing between epithelioid mesothelioma and lung adenocarcinoma. Pathol Int 2007;57:190-9.

18. Abutaily AS, Addis BJ, Roche WR. Immunohistochemistry in the distinction between malignant mesothelioma and pulmonary adenocarcinoma: a critical evaluation of new antibodies. J Clin Pathol 2002;55:662-8.

19. Sandeck HP, Roe OD, Kjaerheim K, et al. Re-evaluation of histological diagnoses of malignant mesothelioma by immunohistochemistry. Diagn Pathol 2010;5:47.

20. Carella R, Deleonardi G, D'Errico A, et al. Immunohistochemical panels for differentiating epithelial malignant mesothelioma from lung adenocarcinoma: a study with logistic regression analysis. Am J Surg Pathol 2001;25:43-50.

21. Yaziji H, Battifora H, Barry TS, et al. Evaluation of 12 antibodies for distinguishing epithelioid mesothelioma from adenocarcinoma: identification of a three-antibody immunohistochemical panel with maximal sensitivity and specificity. Mod Pathol 2006;19:514-23.

22. Klebe S, Nurminen M, Leigh J, et al. Diagnosis of epithelial mesothelioma using tree-based regression analysis and a minimal panel of antibodies. Pathology 2009;41:140-8.

23. Attanoos RL, Webb R, Dojcinov SD, et al. Value of mesothelial and epithelial antibodies in distinguishing diffuse peritoneal mesothelioma in females from serous papillary carcinoma of the ovary and peritoneum. Histopathology 2002;40:237-44.

24. Mawas AS, Amatya VJ, Kushitani K, et al. MUC4 immunohistochemistry is useful in distinguishing epithelioid mesothelioma from adenocarcinoma and squamous cell carcinoma of the lung. Sci Rep 2018;8:134.

25. Xing D, Banet N, Sharma R, et al. Aberrant Pax-8 expression in well-differentiated papillary mesothelioma and malignant mesothelioma of the peritoneum: a clinicopathologic study. Hum Pathol 2018;72:160-6.

26. Le Stang N, Burke L, Blaizot G, et al. Differential Diagnosis of Epithelioid Malignant Mesothelioma With Lung and Breast Pleural Metastasis: A Systematic Review Compared With a Standardized Panel of Antibodies-A New Proposal That May Influence Pathologic Practice. Arch Pathol Lab Med 2019. [Epub ahead of print].

27. Ordóñez NG. Value of calretinin immunostaining in diagnostic pathology: a review and update. Appl Immunohistochem Mol Morphol 2014;22:401-15.

28. Padgett DM, Cathro HP, Wick MR, et al. Podoplanin is a better immunohistochemical marker for sarcomatoid mesothelioma than calretinin. Am J Surg Pathol 2008;32:123-7.

29. Mohammad T, Garratt J, Torlakovic E, et al. Utility of a CEA, CD15, calretinin, and CK5/6 panel for distinguishing between mesotheliomas and pulmonary adenocarcinomas in clinical practice. Am J Surg Pathol 2012;36:1503-8.

30. Lucas DR, Pass HI, Madan SK, et al. Sarcomatoid mesothelioma and its histological mimics: a comparative immunohistochemical study. Histopathology 2003;42:270-9.

31. Klebe S, Brownlee NA, Mahar A, et al. Sarcomatoid mesothelioma: a clinical-pathologic correlation of 326 cases. Mod Pathol 2010;23:470-9.

32. Takeshima Y, Amatya VJ, Kushitani K, et al. Value of immunohistochemistry in the differential diagnosis of pleural sarcomatoid mesothelioma from lung sarcomatoid carcinoma. Histopathology 2009;54:667-76.

33. Tsuji S, Washimi K, Kageyama T, et al. HEG1 is a novel mucin-like membrane protein that serves as a diagnostic and therapeutic target for malignant mesothelioma. Sci Rep 2017;7:45768.

34. Marchevsky AM, LeStang N, Hiroshima K, et al. The differential diagnosis between pleural sarcomatoid mesothelioma and spindle cell/pleomorphic (sarcomatoid) carcinomas of the lung: evidence-based guidelines from the International Mesothelioma Panel and the MESOPATH National Reference Center. Hum Pathol 2017;67:160-8.

35. Ordóñez NG, Sahin AA. Diagnostic utility of immunohistochemistry in distinguishing between epithelioid pleural mesotheliomas and breast carcinomas: a comparative study. Hum Pathol 2014;45:1529-40.

36. Ordóñez NG. Role of immunohistochemistry in distinguishing epithelial peritoneal mesotheliomas from peritoneal and ovarian serous carcinomas. Am J Surg Pathol 1998;22:1203-14. 
37. Amatya VJ, Kushitani K, Mawas AS, et al. MUC4, a novel immunohistochemical marker identified by gene expression profiling, differentiates pleural sarcomatoid mesothelioma from lung sarcomatoid carcinoma. Mod Pathol 2017;30:672-81.

38. Comin CE, Novelli L, Cavazza A, et al. Expression of thrombomodulin, calretinin, cytokeratin 5/6, D2-40 and WT-1 in a series of primary carcinomas of the lung: an immunohistochemical study in comparison with epithelioid pleural mesothelioma. Tumori 2014;100:559-67.

39. Sangoi AR, Fujiwara M, West RB, et al. Immunohistochemical distinction of primary adrenal cortical lesions from metastatic clear cell renal cell carcinoma: a study of 248 cases. Am J Surg Pathol 2011;35:678-86.

40. King JE, Thatcher N, Pickering CA, et al. Sensitivity and specificity of immunohistochemical markers used in the diagnosis of epithelioid mesothelioma: a detailed systematic analysis using published data. Histopathology 2006;48:223-32.

41. Attanoos RL, Dojcinov SD, Webb R, et al. Anti-mesothelial markers in sarcomatoid mesothelioma and other spindle cell neoplasms. Histopathology 2000;37:224-31.

42. Whithaus K, Fukuoka J, Prihoda TJ, et al. Evaluation of napsin A, cytokeratin 5/6, p63, and thyroid transcription factor 1 in adenocarcinoma versus squamous cell carcinoma of the lung. Arch Pathol Lab Med 2012;136:155-62.

43. Provenzano E, Byrne DJ, Russell PA, et al. Differential expression of immunohistochemical markers in primary lung and breast cancers enriched for triple-negative tumours. Histopathology 2016;68:367-77.

44. Ordóñez NG. The diagnostic utility of immunohistochemistry and electron microscopy in distinguishing between peritoneal mesotheliomas and serous carcinomas: a comparative study. Mod Pathol 2006;19:34-48.

45. Pu RT, Pang Y, Michael CW. Utility of WT-1, p63, MOC31, mesothelin, and cytokeratin (K903 and CK5/6) immunostains in differentiating adenocarcinoma, squamous cell carcinoma, and malignant mesothelioma in effusions. Diagn Cytopathol 2008;36:20-5.

46. Lee AH, Paish EC, Marchio C, et al. The expression of Wilms' tumour-1 and Ca125 in invasive micropapillary carcinoma of the breast. Histopathology 2007;51:824-8.

47. Duhig EE, Kalpakos L, Yang IA, et al. Mesothelial markers in high-grade breast carcinoma. Histopathology 2011;59:957-64.

48. Espinosa I, Gallardo A, D'Angelo E, et al. Simultaneous carcinomas of the breast and ovary: utility of Pax8, WT-1, and GATA3 for distinguishing independent primary tumors from metastases. Int J Gynecol Pathol 2015;34:257-65.

49. Müller AM, Franke FE, Muller KM. D2-40: a reliable marker in the diagnosis of pleural mesothelioma. Pathobiology 2006;73:50-4.

50. Carbone M, Shimizu D, Napolitano A, et al. Positive nuclear BAP1 immunostaining helps differentiate nonsmall cell lung carcinomas from malignant mesothelioma. Oncotarget 2016;7:59314-21.

51. Grove A, Paulsen SM, Gregersen M. The value of immunohistochemistry of pleural biopsy specimens in the differential diagnosis between malignant mesothelioma and metastatic carcinoma. Pathol Res Pract 1994;190:1044-55.

52. Comin CE, Novelli L, Boddi V, et al. Calretinin, thrombomodulin, CEA, and CD15: a useful combination of immunohistochemical markers for differentiating pleural epithelial mesothelioma from peripheral pulmonary adenocarcinoma. Hum Pathol 2001;32:529-36.

53. Chaouche-Mazouni S, Scherpereel A, Zaamoum R, et al. Claudin 3, 4, and 15 expression in solid tumors of lung adenocarcinoma versus malignant pleural mesothelioma. Ann Diagn Pathol 2015;19:193-7.

54. Vojtek M, Walsh MD, Papadimos DJ, et al. Claudin-4 immunohistochemistry is a useful pan-carcinoma marker for serous effusion specimens. Cytopathology 2019;30:614-9.

55. Oda T, Ogata S, Kawaguchi S, et al. Immunocytochemical utility of claudin-4 versus those of Ber-EP4 and MOC-31 in effusion cytology. Diagn Cytopathol 2016;44:499-504.

56. Ohta Y, Sasaki Y, Saito M, et al. Claudin-4 as a marker for distinguishing malignant mesothelioma from lung carcinoma and serous adenocarcinoma. Int J Surg Pathol 2013;21:493-501.

57. Ordóñez NG. Value of PAX8, PAX2, claudin-4, and $\mathrm{h}$-caldesmon immunostaining in distinguishing peritoneal epithelioid mesotheliomas from serous carcinomas. Mod Pathol 2013;26:553-62.

58. Facchetti F, Lonardi S, Gentili F, et al. Claudin 4 identifies a wide spectrum of epithelial neoplasms and represents a very useful marker for carcinoma versus mesothelioma diagnosis in pleural and peritoneal biopsies and effusions. Virchows Arch 2007;451:669-80.

59. Jo VY, Cibas ES, Pinkus GS. Claudin-4 immunohistochemistry is highly effective in distinguishing adenocarcinoma from malignant mesothelioma in effusion cytology. Cancer Cytopathol 2014;122:299-306.

60. Chapel DB, Husain AN, Krausz T, et al. PAX8 Expression 
in a Subset of Malignant Peritoneal Mesotheliomas and Benign Mesothelium has Diagnostic Implications in the Differential Diagnosis of Ovarian Serous Carcinoma. Am J Surg Pathol 2017;41:1675-82.

61. Laury AR, Hornick JL, Perets R, et al. PAX8 reliably distinguishes ovarian serous tumors from malignant mesothelioma. Am J Surg Pathol 2010;34:627-35.

62. Ordóñez NG. Value of estrogen and progesterone receptor immunostaining in distinguishing between peritoneal mesotheliomas and serous carcinomas. Hum Pathol 2005;36:1163-7.

63. Berg KB, Churg A. GATA3 Immunohistochemistry for Distinguishing Sarcomatoid and Desmoplastic Mesothelioma From Sarcomatoid Carcinoma of the Lung. Am J Surg Pathol 2017;41:1221-5.

64. Andrici J, Jung J, Sheen A, et al. Loss of BAP1 expression is very rare in peritoneal and gynecologic serous adenocarcinomas and can be useful in the differential diagnosis with abdominal mesothelioma. Hum Pathol 2016;51:9-15.

65. Davidson B, Totsch M, Wohlschlaeger J, et al. The diagnostic role of BAP1 in serous effusions. Hum Pathol 2018;79:122-6.

66. Kapur P, Christie A, Raman JD, et al. BAP1 immunohistochemistry predicts outcomes in a multiinstitutional cohort with clear cell renal cell carcinoma. J Urol 2014;191:603-10.

67. Murali R, Wiesner T, Scolyer RA. Tumours associated with BAP1 mutations. Pathology 2013;45:116-26.

68. Andrici J, Sheen A, Sioson L, et al. Loss of expression of BAP1 is a useful adjunct, which strongly supports the diagnosis of mesothelioma in effusion cytology. Mod Pathol 2015;28:1360-8.

69. Hatem L, McIntire PJ, He B, et al. The role of BRCA1associated protein 1 in the diagnosis of malignant mesothelioma in effusion and fine-needle aspiration cytology. Diagn Cytopathol 2019;47:160-5.

70. Hwang HC, Sheffield BS, Rodriguez S, et al. Utility of BAP1 Immunohistochemistry and p16 (CDKN2A) FISH in the Diagnosis of Malignant Mesothelioma in Effusion Cytology Specimens. Am J Surg Pathol 2016;40:120-6.

71. Cozzi I, Oprescu FA, Rullo E, et al. Loss of BRCA1associated protein 1 (BAP1) expression is useful in diagnostic cytopathology of malignant mesothelioma in effusions. Diagn Cytopathol 2018;46:9-14.

72. Kinoshita Y, Hida T, Hamasaki M, et al. A combination of MTAP and BAP1 immunohistochemistry in pleural effusion cytology for the diagnosis of mesothelioma.
Cancer Cytopathol 2018;126:54-63.

73. Hamasaki M, Kinoshita Y, Yoshimura M, et al. Cytoplasmic MTAP expression loss detected by immunohistochemistry correlates with 9p21 homozygous deletion detected by FISH in pleural effusion cytology of mesothelioma. Histopathology 2019;75:153-5.

74. Churg A, Sheffield BS, Galateau-Salle F. New Markers for Separating Benign From Malignant Mesothelial Proliferations: Are We There Yet? Arch Pathol Lab Med 2016;140:318-21.

75. Nasu M, Emi M, Pastorino S, et al. High Incidence of Somatic BAP1 alterations in sporadic malignant mesothelioma. J Thorac Oncol 2015;10:565-76.

76. Wu D, Hiroshima K, Yusa T, et al. Usefulness of p16/ CDKN2A fluorescence in situ hybridization and BAP1 immunohistochemistry for the diagnosis of biphasic mesothelioma. Ann Diagn Pathol 2017;26:31-7.

77. Hida T, Hamasaki M, Matsumoto S, et al. BAP1 immunohistochemistry and p16 FISH results in combination provide higher confidence in malignant pleural mesothelioma diagnosis: ROC analysis of the two tests. Pathol Int 2016;66:563-70.

78. Hida T, Hamasaki M, Matsumoto S, et al. Immunohistochemical detection of MTAP and BAP1 protein loss for mesothelioma diagnosis: Comparison with 9p21 FISH and BAP1 immunohistochemistry. Lung Cancer 2017;104:98-105.

79. Yoshimura M, Kinoshita Y, Hamasaki M, et al. Diagnostic application of BAP1 immunohistochemistry to differentiate pleural mesothelioma from metastatic pleural tumours. Histopathology 2017;71:1011-4.

80. Shinozaki-Ushiku A, Ushiku T, Morita S, et al. Diagnostic utility of BAP1 and EZH2 expression in malignant mesothelioma. Histopathology 2017;70:722-33.

81. Sheffield BS, Hwang HC, Lee AF, et al. BAP1 immunohistochemistry and p16 FISH to separate benign from malignant mesothelial proliferations. Am J Surg Pathol 2015;39:977-82.

82. Baumann F, Flores E, Napolitano A, et al. Mesothelioma patients with germline BAP1 mutations have 7-fold improved long-term survival. Carcinogenesis 2015;36:76-81.

83. Panou V, Gadiraju M, Wolin A, et al. Frequency of Germline Mutations in Cancer Susceptibility Genes in Malignant Mesothelioma. J Clin Oncol 2018;36:2863-71.

84. Betti M, Aspesi A, Ferrante D, et al. Sensitivity to asbestos is increased in patients with mesothelioma and pathogenic germline variants in BAP1 or other DNA repair genes. Genes Chromosomes Cancer 2018;57:573-83. 
85. Leblay N, Lepretre F, Le Stang N, et al. BAP1 Is Altered by Copy Number Loss, Mutation, and/or Loss of Protein Expression in More Than $70 \%$ of Malignant Peritoneal Mesotheliomas. J Thorac Oncol 2017;12:724-33.

86. Righi L, Duregon E, Vatrano S, et al. BRCA1-Associated Protein 1 (BAP1) Immunohistochemical Expression as a Diagnostic Tool in Malignant Pleural Mesothelioma Classification: A Large Retrospective Study. J Thorac Oncol 2016;11:2006-17.

87. Mashtalir N, Daou S, Barbour H, et al. Autodeubiquitination protects the tumor suppressor BAP1 from cytoplasmic sequestration mediated by the atypical ubiquitin ligase UBE2O. Mol Cell 2014;54:392-406.

88. Bononi A, Giorgi C, Patergnani S, et al. BAP1 regulates IP3R3-mediated $\mathrm{Ca}(2+)$ flux to mitochondria suppressing cell transformation. Nature 2017;546:549-53.

89. Bahnasy AA, El-Din RS, Sabri NA, et al. BAP1 gene mutations in Egyptian patients with advanced sporadic malignant pleural mesothelioma (MPM): relation with clinical outcomes and survival. Cancer Genet 2018;228229:83-92.

90. Cigognetti M, Lonardi S, Fisogni S, et al. BAP1 (BRCA1associated protein 1) is a highly specific marker for differentiating mesothelioma from reactive mesothelial proliferations. Mod Pathol 2015;28:1043-57.

91. Berg KB, Dacic S, Miller C, et al. Utility of Methylthioadenosine Phosphorylase Compared With BAP1 Immunohistochemistry, and CDKN2A and NF2 Fluorescence In Situ Hybridization in Separating Reactive Mesothelial Proliferations From Epithelioid Malignant Mesotheliomas. Arch Pathol Lab Med 2018;142:1549-53.

92. Yoshimura M, Kinoshita Y, Hamasaki M, et al. Highly expressed EZH2 in combination with BAP1 and MTAP loss, as detected by immunohistochemistry, is useful for differentiating malignant pleural mesothelioma from reactive mesothelial hyperplasia. Lung Cancer 2019;130:187-93.

93. Chou A, Toon CW, Clarkson A, et al. The epithelioid BAP1-negative and p16-positive phenotype predicts prolonged survival in pleural mesothelioma.

Histopathology 2018;72:509-15.

94. Hwang HC, Pyott S, Rodriguez S, et al. BAP1 Immunohistochemistry and p16 FISH in the Diagnosis of Sarcomatous and Desmoplastic Mesotheliomas. Am J Surg Pathol 2016;40:714-8.

95. McGregor SM, Dunning R, Hyjek E, et al. BAP1 facilitates diagnostic objectivity, classification, and prognostication in malignant pleural mesothelioma. Hum
Pathol 2015;46:1670-8.

96. Farzin M, Toon CW, Clarkson A, et al. Loss of expression of BAP1 predicts longer survival in mesothelioma. Pathology 2015;47:302-7.

97. Singhi AD, Krasinskas AM, Choudry HA, et al. The prognostic significance of BAP1, NF2, and CDKN2A in malignant peritoneal mesothelioma. Mod Pathol 2016;29:14-24.

98. Walts AE, Hiroshima K, McGregor SM, et al. BAP1 Immunostain and CDKN2A (p16) FISH Analysis: Clinical Applicability for the Diagnosis of Malignant Mesothelioma in Effusions. Diagn Cytopathol 2016;44:599-606.

99. Andrici J, Parkhill TR, Jung J, et al. Loss of expression of BAP1 is very rare in non-small cell lung carcinoma. Pathology 2016;48:336-40.

100. Owen D, Sheffield BS, Ionescu D, et al. Loss of BRCA1associated protein 1 (BAP1) expression is rare in non-small cell lung cancer. Hum Pathol 2017;60:82-5.

101. McGregor SM, McElherne J, Minor A, et al. BAP1 immunohistochemistry has limited prognostic utility as a complement of CDKN2A (p16) fluorescence in situ hybridization in malignant pleural mesothelioma. Hum Pathol 2017;60:86-94.

102. Forest F, Patoir A, Dal Col P, et al. Nuclear grading, BAP1, mesothelin and PD-L1 expression in malignant pleural mesothelioma: prognostic implications. Pathology 2018;50:635-41.

103.Ito T, Hamasaki M, Matsumoto S, et al. p16/CDKN2A FISH in Differentiation of Diffuse Malignant Peritoneal Mesothelioma From Mesothelial Hyperplasia and Epithelial Ovarian Cancer. Am J Clin Pathol 2015;143:830-8.

104. Takeda M, Kasai T, Enomoto Y, et al. 9p21 deletion in the diagnosis of malignant mesothelioma, using fluorescence in situ hybridization analysis. Pathol Int 2010;60:395-9.

105. Chiosea S, Krasinskas A, Cagle PT, et al. Diagnostic importance of 9p21 homozygous deletion in malignant mesotheliomas. Mod Pathol 2008;21:742-7.

106. Wu D, Hiroshima K, Matsumoto S, et al. Diagnostic usefulness of p16/CDKN2A FISH in distinguishing between sarcomatoid mesothelioma and fibrous pleuritis. Am J Clin Pathol 2013;139:39-46.

107.Illei PB, Rusch VW, Zakowski MF, et al. Homozygous deletion of CDKN2A and codeletion of the methylthioadenosine phosphorylase gene in the majority of pleural mesotheliomas. Clin Cancer Res 2003;9:2108-13.

108. Ladanyi M. Implications of P16/CDKN2A deletion in pleural mesotheliomas. Lung Cancer 2005;49 Suppl 1:S95-8. 
109.Krasinskas AM, Bartlett DL, Cieply K, et al. CDKN2A and MTAP deletions in peritoneal mesotheliomas are correlated with loss of p16 protein expression and poor survival. Mod Pathol 2010;23:531-8.

110.Zimling ZG, Jorgensen A, Santoni-Rugiu E. The diagnostic value of immunohistochemically detected methylthioadenosine phosphorylase deficiency in malignant pleural mesotheliomas. Histopathology 2012;60:E96-105.

111. Chapel DB, Schulte JJ, Berg K, et al. MTAP immunohistochemistry is an accurate and reproducible surrogate for CDKN2A fluorescence in situ hybridization in diagnosis of malignant pleural mesothelioma. Mod Pathol 2020;33:245-54.

112. Kinoshita Y, Hamasaki M, Yoshimura M, et al. A combination of MTAP and BAP1 immunohistochemistry is effective for distinguishing sarcomatoid mesothelioma from fibrous pleuritis. Lung Cancer 2018;125:198-204.

113. Rasmussen KD, Helin K. Role of TET enzymes in DNA methylation, development, and cancer. Genes Dev 2016;30:733-50.

114. Vasanthakumar A, Godley LA. 5-hydroxymethylcytosine in cancer: significance in diagnosis and therapy. Cancer Genet 2015;208:167-77.

115. Waterfall JJ, Killian JK, Meltzer PS. The role of mutation of metabolism-related genes in genomic hypermethylation. Biochem Biophys Res Commun 2014;455:16-23.

116. Yang H, Liu Y, Bai F, et al. Tumor development is associated with decrease of TET gene expression and 5-methylcytosine hydroxylation. Oncogene 2013;32:663-9.

117.Lian CG, Xu Y, Ceol C, et al. Loss of 5-hydroxymethylcytosine is an epigenetic hallmark of melanoma. Cell 2012;150:1135-46.

118. Kroeze LI, Aslanyan MG, van Rooij A, et al. Characterization of acute myeloid leukemia based on levels of global hydroxymethylation. Blood 2014;124:1110-8.

119. Roulois D, Deshayes S, Guilly MN, et al. Characterization of preneoplastic and neoplastic rat mesothelial cell lines: the involvement of TETs, DNMTs, and 5-hydroxymethylcytosine. Oncotarget 2016;7:34664-87.

120. Chapel DB, Husain AN, Krausz T. Immunohistochemical evaluation of nuclear 5-hydroxymethylcytosine $(5-\mathrm{hmC})$ accurately distinguishes malignant pleural mesothelioma from benign mesothelial proliferations. Mod Pathol 2019;32:376-86.

121. Comet I, Riising EM, Leblanc B, et al. Maintaining cell identity: PRC2-mediated regulation of transcription and cancer. Nat Rev Cancer 2016;16:803-10.
122.LaFave LM, Beguelin W, Koche R, et al. Loss of BAP1 function leads to EZH2-dependent transformation. Nat Med 2015;21:1344-9.

123.Kemp CD, Rao M, Xi S, et al. Polycomb repressor complex-2 is a novel target for mesothelioma therapy. Clin Cancer Res 2012;18:77-90.

124.Jiang H, Gupta R, Somma J. EZH2, a unique marker of malignancy in effusion cytology. Diagn Cytopathol 2014;42:111-6.

125. Galateau Salle F, Le Stang N, Nicholson AG, et al. New Insights on Diagnostic Reproducibility of Biphasic Mesotheliomas: A Multi-Institutional Evaluation by the International Mesothelioma Panel From the MESOPATH Reference Center. J Thorac Oncol 2018;13:1189-203.

126.Arzt L, Quehenberger F, Halbwedl I, et al. BAP1 protein is a progression factor in malignant pleural mesothelioma. Pathol Oncol Res 2014;20:145-51.

127.Pulford E, Huilgol K, Moffat D, et al. Malignant Mesothelioma, BAP1 Immunohistochemistry, and VEGFA: Does BAP1 Have Potential for Early Diagnosis and Assessment of Prognosis? Dis Markers 2017;2017:1310478.

128. Pastorino S, Yoshikawa Y, Pass HI, et al. A Subset of Mesotheliomas With Improved Survival Occurring in Carriers of BAP1 and Other Germline Mutations. J Clin Oncol 2018. [Epub ahead of print].

129. Hamasaki M, Matsumoto S, Abe S, et al. Low homozygous/high heterozygous deletion status by p16 FISH correlates with a better prognostic group than high homozygous deletion status in malignant pleural mesothelioma. Lung Cancer 2016;99:155-61.

130. Dacic S, Kothmaier H, Land S, et al. Prognostic significance of $\mathrm{p} 16 / \mathrm{cdkn} 2 \mathrm{a}$ loss in pleural malignant mesotheliomas. Virchows Arch 2008;453:627-35.

131. Khanna S, Thomas A, Abate-Daga D, et al. Malignant Mesothelioma Effusions Are Infiltrated by CD3(+) T Cells Highly Expressing PD-L1 and the PD-L1(+) Tumor Cells within These Effusions Are Susceptible to ADCC by the Anti-PD-L1 Antibody Avelumab. J Thorac Oncol 2016;11:1993-2005.

132. Combaz-Lair C, Galateau-Salle F, McLeer-Florin A, et al. Immune biomarkers PD-1/PD-L1 and TLR3 in malignant pleural mesotheliomas. Hum Pathol 2016;52:9-18.

133. Nguyen BH, Montgomery R, Fadia M, et al. PD-L1 expression associated with worse survival outcome in malignant pleural mesothelioma. Asia Pac J Clin Oncol 2018;14:69-73.

134.Inaguma S, Lasota J, Wang Z, et al. Expression of ALCAM 
(CD166) and PD-L1 (CD274) independently predicts shorter survival in malignant pleural mesothelioma. Hum Pathol 2018;71:1-7.

135. Cedrés S, Ponce-Aix S, Zugazagoitia J, et al. Analysis of expression of programmed cell death 1 ligand 1 (PD-L1) in malignant pleural mesothelioma (MPM). PLoS One 2015;10:e0121071.

136. Cedrés S, Ponce-Aix S, Pardo-Aranda N, et al. Analysis of expression of PTEN/PI3K pathway and programmed cell death ligand 1 (PD-L1) in malignant pleural mesothelioma (MPM). Lung Cancer 2016;96:1-6.

137. Brosseau S, Danel C, Scherpereel A, et al. Shorter Survival in Malignant Pleural Mesothelioma Patients With High PD-L1 Expression Associated With Sarcomatoid or Biphasic Histology Subtype: A Series of 214 Cases From the Bio-MAPS Cohort. Clin Lung Cancer 2019;20:e564-75.

138. Chapel DB, Stewart R, Furtado LV, et al. Tumor PDL1 expression in malignant pleural and peritoneal mesothelioma by Dako PD-L1 22C3 pharmDx and Dako PD-L1 28-8 pharmDx assays. Hum Pathol 2019;87:11-7.

139. Forde PM, Scherpereel A, Tsao AS. Use of Immune Checkpoint Inhibitors in Mesothelioma. Curr Treat Options Oncol 2019;20:18.

140.Henderson DW, Shilkin KB, Whitaker D. Reactive mesothelial hyperplasia vs mesothelioma, including mesothelioma in situ: a brief review. Am J Clin Pathol 1998;110:397-404.

141. Cury PM, Butcher DN, Corrin B, et al. The use of histological and immunohistochemical markers to

Cite this article as: Chapel DB, Schulte JJ, Husain AN, Krausz T. Application of immunohistochemistry in diagnosis and management of malignant mesothelioma. Transl Lung Cancer Res 2020;9(Suppl 1):S3-S27. doi: 10.21037/tlcr.2019.11.29 distinguish pleural malignant mesothelioma and in situ mesothelioma from reactive mesothelial hyperplasia and reactive pleural fibrosis. J Pathol 1999;189:251-7.

142. Churg A, Hwang H, Tan L, et al. Malignant mesothelioma in situ. Histopathology 2018;72:1033-8.

143. Churg A, Galateau-Salle F, Roden AC, et al. Malignant mesothelioma in situ: morphologic features and clinical outcome. Mod Pathol 2020;33:297-302.

144. Allen TC, Cagle PT, Churg AM, et al. Localized malignant mesothelioma. Am J Surg Pathol 2005;29:866-73.

145. Gelvez-Zapata SM, Gaffney D, Scarci M, et al. What is the survival after surgery for localized malignant pleural mesothelioma? Interact Cardiovasc Thorac Surg 2013;16:533-7.

146. Butnor KJ, Pavlisko EN, Sporn TA, et al. Mesothelioma of the tunica vaginalis testis. Hum Pathol 2019;92:48-58.

147.Jones MA, Young RH, Scully RE. Malignant mesothelioma of the tunica vaginalis. A clinicopathologic analysis of 11 cases with review of the literature. Am J Surg Pathol 1995;19:815-25.

148. Winstanley AM, Landon G, Berney D, et al. The immunohistochemical profile of malignant mesotheliomas of the tunica vaginalis: a study of 20 cases. Am J Surg Pathol 2006;30:1-6.

149. Ortiz-Villalón C, Le Stang N, Galateau Salle F, et al. Pericardial Mesothelioma: A Multi-institutional Study of 61 cases. In: United States and Canadian Academy of Pathology. National Harbor, Maryland, USA, 2019. 